RESEARCH

IN

\title{
APPLIED GEOPHYSICS
}

No. 31

1984

Electromagnetic Induction in an Inhomogeneous Conductive Thin Sheet Richard S. Smith

GEOPHYSICS LABORATORY DEPARTMENT OF PHYSICS UNIVERSITY OF TORONTO 


\title{
ELECTROMAGNETIC INDUCTION \\ IN AN INHOMOGENEOUS \\ CONDUCTIVE THIN SHEET
}

by

\author{
Richard Smith \\ Geophysics Laboratory \\ Department of Physics \\ University of Toronto
}

\begin{abstract}
A thesis submitted in conformity with the requirements for the degree of Master of Science in the University of Toronto
\end{abstract}
(C) 1984
by Richard Smith 

ERRATA

(1) Although the program PLATE is consistent with the results of the frequency domain method (section 3.2.1), scale model studies agree even more closely.

(2) The vertical magnetic field is seen to increase by two orders of magnitude on figures 4.38 and 4.39 (discussed on page 44 lines 23-25). These increases are due to coding errors in the computer program. The errors from numerical sources can thus be contained by filtering the field and expanding the grid.

(3) The calculations are presently done on an array processor and run times are of the order of 10 to 15 minutes. This allows filtering/expansion decisions to be made interactively. 



\section{ABSTRACI}

In electrically conductive geological environments electromagnetic prospecting systems experience difficulty in sensing bodies buried below conductive overburden. Not only may coupling exist between the conductor and the overburden, but in many cases an anomalous response may be due entirely to lateral variations in the conductivity of the overburden.

This thesis presents a method of calculating the electromagnetic response of a thin sheet with a laterally varying conductivity-thickness product. The thin sheet is suspended in free space. This model will approximate the effect of an overburden layer lying on an unweathered resistive crystaline rock.

Two solution methods were used. The first was a frequency domain method in which a matrix equation was solved to find the horizontal wavenumber components of the induced vertical magnetic field. The method is best suited to calculating the response of the sheet when excited by frequency domain airborne E.M. systems.

The second method calculates the time domain step-response of the sheet by time stepping the component of the magnetic field which cuts normally through the sheet. To calculate the time derivative of the normal (vertical) field, the horizontal 
components of the magnetic field must be known. The conversion of the vertical components of the magnetic field to the horizontal components of the magnetic field is done in the wavenumber domain. To transform the field to wavenumber domain the fast Eourier transform is used.

The time domain method works well when the sheet is excited by a large loop placed a small distance above the sheet. The conductance of the sheet must vary smoothly. Instabilities result at late times, but these have been overcome by forcing the solution towards the solution for a uniform thin sheet. 


\section{ACKNOWLEDGEMENTS}

Eirstly I would like to thank my thesis supervisor Professor G.E. West for the invaluable suggestions he made regarding the subject matter of this thesis.

I would also like to thank Professor R.N. Edwards. It was he who first suggested this topic. He also provided the Eortran subroutine for calculating the fast Eourier transform.

Professor R.C. Bailey correcting some of my misconceptions concerning Eourier transforms, while Professor W.R. Peltier guided me in the methods of time stepping fields.

Discussions I had with A.B. Ferneyhough concerning the more practical aspects of helicopter E.M. were helpful. Also Ben Polzer, Peter Walker and Ross Groom must be thanked, as they showed an interest in this topic, and were more than happy to devote some thought to the subject matter and make intelligent suggestions.

Special thanks must go to Ross Groom for supplying the Gaussian Elimination routine which was modified for use in this project. The interactive terminal plotting routine 'TPLOT' which was written by Dave Boerner was used extensively throughout the project, and Dave deserves a great deal of credit for this piece of software.

The contour plots were generated using a routine written by the National Centre for Atmospheric Research, Boulder Colorado. 
The cost of my computing and photocopying etc was covered by a National Science and Engineering Research Council (NSERC) Grant to Prof. G.E. West, and for this due acknowledgement must be given.

My own support was provided by the Association of Universities and Colleges of Canada through a Bursary granted under the auspices of the British Commonwealth Scholarship and Eellowship Plan.

Einally I would like to thank all those who are reading this acknowledgement, as what use would a thesis be if it was never read! 
Abstract

Acknowledgements

Table of contents

1.0 CHAPTER $1-$ INTRODUCTION $\ldots \ldots \ldots \ldots \ldots \ldots$

2.0 CHAPTER 2 -INDUCTION IN THIN SHEETS......... 5

2.1 Potential Eunctions..................... 8

2.2 Eourier Domain.........................

2.3 Relations Between Quantities In Wavenumber Domain... 11

3.0 CHAPTER 3 - THE EREOUENCY DOMAIN SOLUTION....... 13

3.1 The Constant Conductivity Case................ 15

3.2 The Variable Conductivity Case................. 19

3.2 .1 Numerical Checks...................... 20

4.0 CHAPTER 4 - TIME DOMAIN SOLUTION ........... 22

4.I The Constant Conductivity Case.............. 23 
4.2 Methods of Time stepping.................. 30

4.3 The Variable Conductivity Case .............. 37

4.3 .1 Model Runs.......................... 40

4.3.1.1 Checks Against The Uniform Sheet Case.......... 40

4.3 .1 .2 A Resistive Inhomogeneity................ 40

4.3 .1 .3 A Conductive Inhomogeneity............... 42

4.4 Accuracy Tests........................ 42

4.4 .1 Internal Consistency Checks............... 43

4.4 .1 .1 Grid Spacing....................... 43

4.4 .1 .2 Time step size.................... 45

5.0 CHAPTER 5 - CONLUSION ................ 46

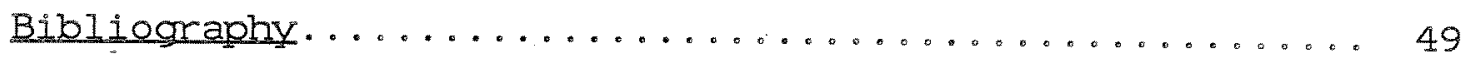




\subsection{CHAPTER 1 - INTRODUCTION}

Ore bodies are generally more conductive than the surrounding rock, and therefore it is possible to detect their presence using electromagnetic (E.M.) prospecting systems. In terrains such as Australia and South Africa a weathered surface layer (overburden) will frequently be more conductive than the surrounding bedrock. It is sometimes not possible to distinguish the E.M. response of an overburden with a laterally varying conductivity from the E.M. response of a conductor (ore body) buried beneath an overburden. This thesis presents a method of calculating the E.M. response of an overburden with laterally varying conductivity. This will allow overburdens of non-uniform conductivity to be modelled, and hopefully it will enable non-uniform overburdens to be distinguished from buried conductors.

Man-made or artificial source E.M. prospecting systems consist of a transmitter and a receiver (for general reference see Grant and West (1965), Ward (1967) and Telford et al (1976)). The transmitter transmits a time-varying primary magnetic field which induces currents in conductive target bodies. A secondary magnetic field is associated with the induced currents, and the receiver measures this field (or a quantity related to $i t)$. Results are generally displayed by plotting the secondary field along profiles, and early interpretation techniques mostly involved the recognition of anomalous "bumps" on these profiles. The anomalies indicate lateral changes in the secondary field and therefore lateral 
changes in the conductivity of the target zone.

Erequency domain systems measure the response of the target when it is excited by a primary magnetic field whose magnitude is oscillating at a fixed frequency (or a number of fixed frequencies). The nature of the currents induced in the ground varies with the frequency of the exciting field. Therefore exciting the ground with several frequencies results in more information being obtained about the conductivity structure of the ground. For example, if a conducting body exhibits an anomalous response both at a high frequency and at a low frequency, it must be a better conductor than one which only has an anomalous response at a high frequency. If the conductor is excited with several frequencies, then even more information relating to the target body can be obtained. An extension of this concept leads to taking measurements in the time domain. This involves taking measurements of the secondary field as a function of delay time (the time after the earth has been pulsed with a magnetic field). An early example of a time domain system is the INPUT system (See Barringer (1962) or Nelson and Morris (1969) for a description of the system).

Frequency and time domain E.M. systems can be divided into two categories - moving transmitter and fixed transmitter systems. Eor logistic reasons the moving transmitter systems are light in weight, and generally take measurements in the frequency domain. The fixed transmitter systems are larger and heavier (large transmitter loops), and generally take measurements in the time domain. For the moving transmitter systems the distance between the transmitter and receiver is generally fixed, whilst for the fixed transmitter systems the 
relative position of the receiver is varied.

Geophysical measurements are made so that the form and physical properties of an unknown structure can be infered from the measured response. This is done by assuming that a conductor takes a certain form. The response associated with that form is then calculated, and the parameters which describe the body (e.g. size, orientation, conductivity, depth, etc.) are adjusted until the calculated response closely matches the measured response. The bodies which can be modelled are restricted to those for which the E.M. response can be calculated.

Responses have been obtained using scale model studies, theoretical solutions and computer models. Examples of scale model results are those published by Palacky (1975) and Gupta et al (1980). Theoretical solutions have been obtained for an infinite cylinder (Verma (1972)), a sphere in a conductive medium (Singh (1973)), a layered earth (Knight and Raiche (1983)), and many other examples to be found in the geophysical literature. Numerical computer models have also been obtained, and examples of these are Wannamaker et al (1984), Das and Verma (1982) and Lajoie and West (1976).

A large number of the theoretical and computer models assume that the conductive body behaves as if it were situated in free space (for example Mallick (1972) and Dyck et al (1980)). In many cases this is an adequate assumption, however in other cases it is not, and even if the host medium is very resistive, the target zone may be overlain by a conducting overburden. In cases such as this electromagnetic coupling 
makes the response more difficult to calculate. Scale modelling with conductive overburdens has been done by Lowrie and West (1965) and Gaur and Verma (1973). Oristaglio and Hohmann (1984) have provided a numerical solution to the problem of a conductor of arbitrary shape beneath a conducting overburden by stepping the fields through time. They assumed that the fields vary only in two dimensions. The source must therefore be one or more infinite wires, and the body and overburden must extend for an infinite distance along strike.

Methods of computing the E.M. response due to a laterally varying sheet (overburden) have been found in the case of the magneto-telluric method (recent examples are Greenfield (1971) and MCKirdy and Weaver (1984)). Eor a finite three dimensional source Geyer and Wait (1978) obtained the E.M. response for a laterally varying conducting sheet, however their method is restricted to variations which have cylindrical symmetry.

In Chapter 2 of this thesis equations have been derived which can be used to calculate the response when an arbitrary source excites a sheet of arbitrary conductivity distribution (within certain limits). The frequency domain solution is found in Chapter 3, and the method is best suited to moving transmitter loops which are some distance above the sheet (such as in airborne systems). Time domain systems generally have large transmitter loops lying on the ground. In Chapter 4 a method has been developed which can calculate the decay of the fields associated with such loops by stepping the fields through time using a finite difference approximation. 


\subsection{CHAPTER 2 - INDUCTION IN THIN SHEETS}

The problem of Electomagnetic induction in thin sheets of infinite extent has been examined by Price (1949). The theory, as outlined here, follows Price (1949). Grant and West (1965) and Edwards (unpublished 1973).

Consider a sheet of infinite extent, which for convenience can be considered to be lying in the $z=0$ plane of a Cartesian co-ordinate system. The sheet has a thickness ' $d$ ' which is assumed to be very small, but the conductivity 0 is so large that $\sigma d$ remains finite as $d$ vanishes. Define the 'resistance' $R(x, y)$ where

$$
R(x, y)=1 \cdot /(\sigma(x, y) d(x, y))
$$

The currents induced in the sheet will be contained entirely within the sheet, and thus we can define a surface current density Khere

$$
\mathrm{K}=\mathrm{I} d=\mathrm{E} \text { od }=\mathrm{E} / \mathrm{R} \text {. }
$$

Here $E$ is the total electric field intensity. Once again I can be infinite, but $K$ must remain finite.

The total magnetic field intensity may be divided up into two components i.e.

$$
\mathrm{H}=\mathrm{H}^{\mathrm{e}}+\mathrm{H}^{i}
$$

where $H^{e}$ is the primary component of the field which excites the 
sheet and $\mathrm{H}^{i}$ is the secondary component associated with the currents induced in the sheet. These shall be refered to as the exciting and induced fields respectively.

The boundary conditions which apply to the magnetic fields in the vicinity of the sheet are:

(i) the exciting field (H) must be continuous through the sheet,

(ii) the normal component of the induced field $\left(\mathrm{H}_{\varkappa}^{i}\right)$ must be continuous through the sheet, and

(iii) the tangential components $\left(\mathrm{H}_{x}^{i}\right.$ and $\left.\mathrm{H}_{y}^{i}\right)$ of the induced magnetic field will be equal in magnitude and opposite in sign on the positive $(+)$ and negative $(-)$ sides of the sheet. The last boundary condition is a function of the symmetry of the induced field about the sheet (see Grant and West (1965) for further details). The boundary conditions can be written mathematicaliy as follows:

$$
\begin{array}{r}
\mathrm{H}_{t}^{\mathrm{e}}-\mathrm{H}_{0}^{\mathrm{e}}=0 \\
\mathrm{n} \cdot\left[\mathrm{H}_{t}^{i}-\mathrm{H}_{-}^{i}\right]=0 \\
\mathrm{n} \times\left[\mathrm{H}_{t}^{i}+\mathrm{H}_{0}^{\delta}\right]=0
\end{array}
$$

where the + and the - represent the flelds above and below the sheet respectively.

Assuming no displacement currents, Maxwell's equations and Ohm's law can be written as follows:

$$
\operatorname{div}(\varepsilon E)=98
$$




$$
\begin{aligned}
& \operatorname{div}(\mu \mathrm{H})=0 \\
& \operatorname{cur} 1(\mathrm{H})=\underline{J} \\
& \operatorname{curl}(\mathrm{E})=-\mu \mathrm{dH} / \mathrm{dt}
\end{aligned}
$$

and

$$
I=O E,
$$

where

$$
\begin{aligned}
& \mu=\text { the magnetic permeability of the media, } \\
& \varepsilon=\text { the electric permittivity of the media, } \\
& \rho_{\xi}=\text { the charge density of the free charges and } \\
& t=\text { time. }
\end{aligned}
$$

The normal (z) component of 2.0 .10 can be written

$$
\frac{\partial E_{y}}{\partial x}-\frac{\partial E_{x}}{\partial y}=-\mu \frac{\partial H_{z}}{\partial t}
$$

Because the currents flows entirely within the sheet, 2.0 .11 gives:

$$
\begin{aligned}
& E_{x}=R K_{x} \\
& E_{y}=R K_{y}
\end{aligned}
$$

Expanding 2.0.9 and using the boundary conditions 2.0 .4 to 2.0 .6 yields:

$$
\begin{aligned}
& \mathrm{K}_{x}=-2 \mathrm{H}_{y+}^{i} \\
& \mathrm{~K}_{y}=2 \mathrm{H}_{x_{+}}^{i}
\end{aligned}
$$


Substitute 2.0 .13 to 2.0 .16 into 2.0 .12

$$
\mathrm{R} \operatorname{div}\left(\mathrm{H}_{+}^{i}\right)+\operatorname{grad}(\mathrm{R}) \cdot \mathrm{H}_{+}^{i}=-\frac{\mu}{2} \frac{\partial \mathrm{H}_{2}}{\partial t}
$$

where div and grad are two-dimensional operators in the $z=0$ plane. This differential equation must be solved to find the induced fields on the positive side of the sheet.

\section{I Rotential Functions}

If we assume that $\mu$ is constant throughout the medium, then $\operatorname{div}(\mathrm{H})=0$ (from 2.0.8). Outside the sheet (in the dielectric) cur $(\mathrm{H})=0$, and thus we can define a Magnetic potential $\phi$ such that

$$
\mathrm{H}=-\operatorname{grad}(\phi)
$$

and $\phi$ will be a solution to Laplace's equation.

As the problem is linear the potential $\phi$ can be divided into exciting and induced potentials.

$$
\begin{aligned}
& \mathrm{H}_{+}^{\mathrm{e}}=-\operatorname{grad}\left(\phi_{+}^{e}\right) \\
& \mathrm{H}_{+}^{i}=-\operatorname{grad}\left(\phi_{t}^{i}\right) .
\end{aligned}
$$

In the sheet $\mathrm{K}$ is divergence free, and thus it can be represented by the curl of a scalar stream potential.

$$
\underline{K}=\operatorname{cur} I\left(\Omega \Psi^{\prime \prime}\right)
$$




$$
=-n \times \operatorname{grad}(\psi)
$$

but from 2.0 .15 and 2.0 .16

$$
\begin{aligned}
\mathrm{K} & =\mathrm{n} \times 2 \mathrm{H}_{+}^{i} \\
& =-\mathrm{n} \times 2 \operatorname{grad}\left(\phi_{+}^{i}\right)
\end{aligned}
$$

thus

$$
y=2 \phi^{i}
$$

All the quantities are measured on the positive side of the sheet, and thus the + subscript can be dropped.

\subsection{Eourier Domain}

If $f(x, y)$ is a two dimensional function in the space domain, then the two dimensional transform $\vec{E}(p, q)$ and the function are connected via the relations (see Bracewell (1978)):

$$
\begin{aligned}
& \vec{E}(p, q)=\int_{-\infty}^{\infty} \int_{-\infty}^{\infty} f(x, y) \exp (-i p x-i q y) d x d y \\
& f(x, y)=(1 /(2 \pi))^{2} \int_{-\infty}^{\infty} \int_{-\infty}^{\infty} \vec{E}(p, q) \exp (+i p x+i q y) d p d q
\end{aligned}
$$

where i is the square root of negative one. The short hand notation for the relation between Eourier transform pairs is:

$$
f(x, y) \quad-\cdots \vec{E}(p, q)
$$

The two dimensional convolution of two functions (represented by 素) can be written: 


$$
f(X, Y)=\int_{-\infty}^{\infty} g(x, y)+\infty(x-\xi, Y-\eta) g(\xi, \eta) d \xi d \eta
$$

Using the convolution theorem,

$$
f(x, y) \text { 䖭 } g(x, y) \longleftrightarrow-\cdots \bar{E}(p, q) \bar{G}(p, q)
$$

While if

$$
\overline{\mathrm{F}}(p, q) \text { * } \overline{\mathrm{G}}(p, q)=\int_{-\infty}^{\infty} \int_{-\infty}^{\infty} \overline{\mathrm{E}}(p-\xi, q-\eta) \overline{\mathrm{G}}(\xi, \eta) d \xi d \eta .
$$

then,

$$
\bar{E}(p, q) \bar{G}(p, q) \leftrightarrow(2 \pi)^{2} \quad f(x, y) g(x, y)
$$

The differentiation rules are:

$$
\begin{aligned}
& d(f(x, y)) / d x<-\cdots>\text { i } p \bar{E}(p, q) \\
& d(f(x, y)) / d y<-\cdots+i q \bar{E}(p, q) \text {. }
\end{aligned}
$$

If $f(x, y)$ satisfies Laplace's equation, and has a transform $\vec{E}(p, q)$, then:

$$
\left(d^{2} / d x^{2}+d^{2} / d y^{2}+d^{2} / d z^{2}\right) f(x, y)=0
$$

becomes, on transforming to the wave-number domain:

$$
-p^{2} \bar{E}(p, q)-q^{2} \bar{E}(p, q)=-d^{2}(\bar{E}(p, q)) / d z^{2} .
$$

The solution to this differential equation is: 


$$
\left.\bar{E}(p, q)\right|_{z=z^{\prime}}=\left.\bar{E}(p, q)\right|_{z=0} \exp \left( \pm z^{\prime}\left(p^{2}+q^{2}\right)^{1 / z}\right)
$$

Where the \pm sign depends on whether $z=z^{*}$ is nearer $(+)$ or farther $(-)$ than $z=0$ from the source.

$$
\begin{aligned}
& \text { Erom }(2.2 .3) \text { we can write: } \\
& d(\vec{E}(p, q)) /\left.d z\right|_{z=z^{\prime}}= \pm\left.\left(p^{2}+q^{2}\right)^{1 / 2} \vec{E}(p, q)\right|_{z=z^{\prime}}
\end{aligned}
$$

and

$$
d^{2}(\bar{E}(p, q)) /\left.d z^{2}\right|_{z=z^{\prime}}=\left.\left(p^{2}+q^{2}\right) \quad \bar{E}(p, q)\right|_{z=z^{\prime}}
$$

\subsection{Relations between quantities in Wavenumber domain}

The vertical and horizontal components of the field are the negative vertical and horizontal derivatives of the scalar potential. Therefore they are related to each other in the Eourier domain.

Erom 2.1 .1 and 2.1 .4 the Eourier transform of the vertical component of the magnetic field and the stream potential are related as follows:

$$
\vec{\psi}(p, q)=\frac{2 H_{z}^{i}(p \cdot q, O)}{\left(p^{2}+q^{2}\right)^{1 / 2}} \quad\left(p^{2}+q^{2}\right)=0
$$

Eor $p=q=0$, the $\overline{\mathrm{H}}_{z}^{i}$ and $\bar{\psi}$ can be set to zero. The magnitude of the zero wavenumber term is not important, as it transforms to a constant value in space domain, and only the derivatives of the stream potential are important. The lines of constant $\psi(x, y)$ represent flow directions for the current. 
The transforms of the vertical and horizontal fields are thus related as follows:

$$
\vec{H}_{x}^{i}(p, q, z)=-i p \frac{\vec{H}_{z}^{i}(p, q, 0)}{\left(p^{3}+q^{2}\right)^{1 / 2}} \exp \left(-z\left(p^{2}+q^{2}\right)^{1 / z}\right)
$$

similarly

$$
\vec{H}_{y}^{i}(p, q, z)=-i q \frac{\vec{H}_{z}^{i}(p, q, o)}{\left(p^{2}+q^{2}\right)^{1 / 2}} \exp \left(-z\left(p^{2}+q^{2}\right)^{1 / 2}\right)
$$

To obtain the space domain fields, these must be inverse Eourier trans formed. 


\subsection{CHAPTER 3 - THE EREQUENCY DOMAIN SOLUTION}

The Eourier transform of 2.0 .17 is:

$\left[\overrightarrow{\mathrm{R}}\left(\mathrm{p}^{2}+\mathrm{q}^{2}\right)^{1 / 2}+\mathrm{pR} \frac{\mathrm{p}}{\left(\mathrm{p}^{2}+\mathrm{q}^{2}\right)^{1 / 2}}+q \overrightarrow{\mathrm{R}} \frac{q}{\left(\mathrm{p}^{2}+\mathrm{q}^{2}\right)^{2 / 2}}\right] \overrightarrow{\mathrm{H}}_{\mathrm{z}}^{i}+2 \pi^{2} \mu i w \mathrm{H}_{\mathrm{z}}^{i}$

$$
=-2 \pi^{2} \mu \dot{i} \omega \tilde{H}_{Z}^{e}
$$

where $\overline{\mathrm{R}}(p, q)$ and $\overline{\mathrm{H}}_{2}^{\mathrm{Z}}(p, q)$ are known, and an exp (iwt) time dependence is assumed ( $\omega$ is frequency in $\mathrm{rad} / \mathrm{sec}$ ).

If the discrete value Eourier transform is used, then $\bar{H}_{2}^{e}(p, q)$ and $\bar{H}_{2}^{i}(p, q)$ are vectors, and thus 3.0 .1 is a matrix equation of the form

$$
\{A+c(\omega) I\} \vec{H}_{z}^{i}(p, q)=\bar{H}_{z}^{e}(p, q) \text {. }
$$

where $A$ is a complex matrix, $c$ is a constant (for fixed $\omega$ ) and $I$ is the identity matrix. For a specified resistance distribution and frequency $\{A+C I\}$ can be inverted, and the inverse used to solve for many different right hand side vectors. Each different right hand side vector will represent the transform of the exciting field for a different transmitter position, and hence profiles such as those which are normally obtained in Helicopter EM (HEM) work can be calculated.

Implicit in the use of the discrete Eourier transform is the assumption that the transformed field repeats itself periodically in space. The exciting source is therefore made up of many identical sources which repeat periodically in the space domain. If the source fields do not approximate to zero at the edge of the grid, then the effect from sources outside the 
sampled grid will not be negligible. Thus care must be taken to ensure that regions where the exciting field is significant do not approach too closely to the edge of the discretization grid.

A complex matrix solver is required to invert $\{\AA+c I\}$. of the solvers tested, the most efficient was a Gaussian elimination routine due to Groom (pers. com. 1984), which operates on columns and uses partial pivoting.

The discretization of the resistance function and the magnetic fields must be identical. To represent the fields well. they must be sampled closely to avoid aliasing errors, and sufficiently far out that the fields become negligible at the end of the grid. This results in a grid with a large number of nodes, and the resulting matrix equation will therefore be large. For example, if there are 128 sample intervals in the $x$ direction and 128 sample intervals in the $y$ direction, then there will be 16384 wavenumbers in $(p, q)$ space. Thus a 16384 by 16384 complex matrix must be inverted to solve the system of equations. Obviously this is prohibitive. If the assumed source is a Helicopter EM system, then the exciting field will be band limited. If the resistance variation is reasonably smooth, then the induced fields upward continued to the level of the receiver should also be band limited. The fields can therefore be represented with sufficient accuracy by a small number of wavenumbers. Therefore it will not be necessary to solve for all the wavenumbers, and this will save computer time.

Decreasing the number of wavenumbers used to represent the resistance function means that the convolutions on the left hand side of 3.0 .1 can be done in less computer time. This has an 
effect of smoothing the resistance function. The smoothing operation may or may not be representative of the geological situation being modelled.

\subsection{The Constant conductivity Case}

Grant and West (1965). Geyer and Wait (1978) and Wait (1982) have solved the problem of a thin sheet of constant resistance excited by a dipole source. When the resistance is constant, only the zero wavenumber term is non-zero in $\overline{\bar{R}}(p, q)$. The convolutions drop out (as do the $2 \pi^{2}$ factors) and 3.0 .1 reduces to the trivial relation (Edwards unpublished 1973):

$$
\vec{H}_{z}^{i}(p, q)=\frac{-\mu i \omega \vec{H}_{z}^{e}(p, q)}{2 R\left(p^{2}+q^{2}\right)^{1 / 2} \mu i \omega} .
$$

This is Maxwell's retreating image solution written in $(p, q)$ domain.

Wait's solution has been used to check the accuracy of the Eourier domain solution presented here. The transmitting and receiving dipoles are assumed to be vertical.

Eigure 3.1 shows the secondary vertical fields excited by a vertical dipole oscillating at $300 \mathrm{~Hz} 30$ metres above a plate of constant conductivity thickness product ( $R=0.01 \mathrm{Ohms}$ ). The designation 'Wait' is used to represent the solution obtained using the method of Geyer and Wait. The designation 'Eourier' is used to designate the Fourier domain solution discussed in this chapter. In this case a 128 by 128 grid with spacing between the nodes of 4 metres has been selected. 


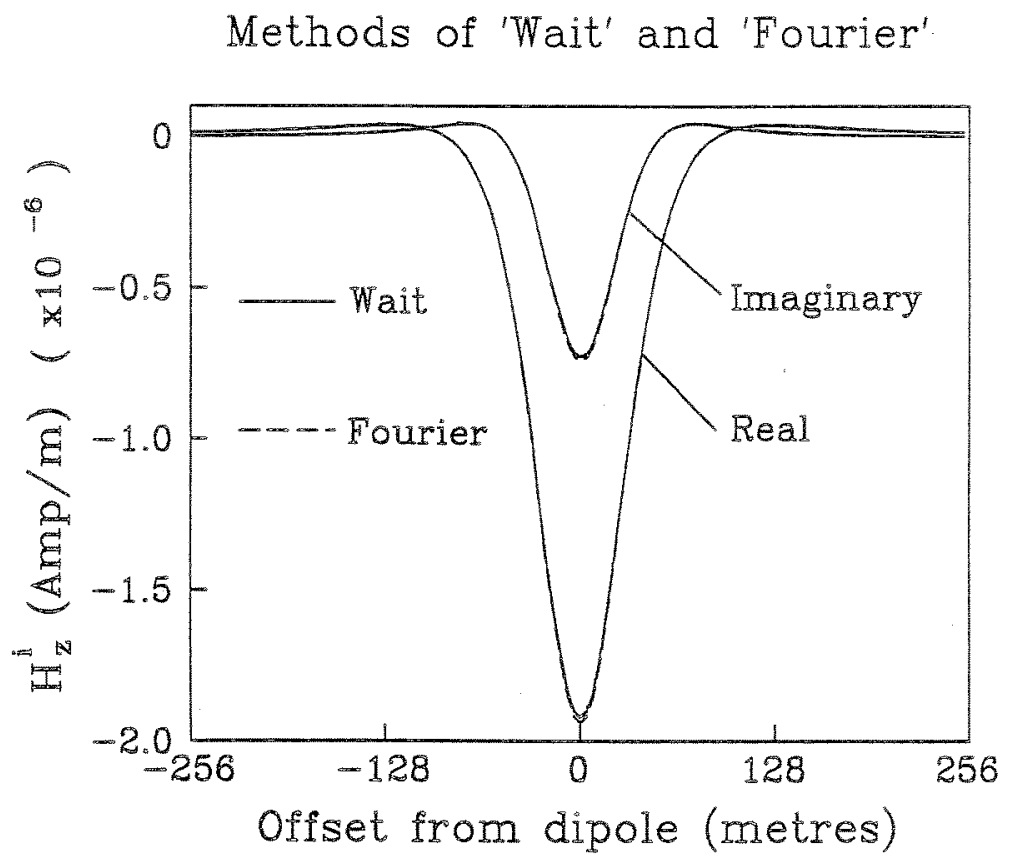

EICURE 3.1 comparison of the solution methods of waite and the Eourler domain case discussed in this chapter. The secondary vertical fields are plotted as a function of distance from a vertical exciting dipole oscillating at 300 Hiz 30 metres above a plate of constant conductivity thickeness product ( $0 t=100$ Siemens). The Eourter method discretization orid stretches from $=256$ to 252 in the $x$ and $y$ directions. The spacing between nodes is 4 metres in both directions.

Induced Response: dipole sep $=9 \mathrm{~m} ; \mathrm{h}=30 \mathrm{~m}$.

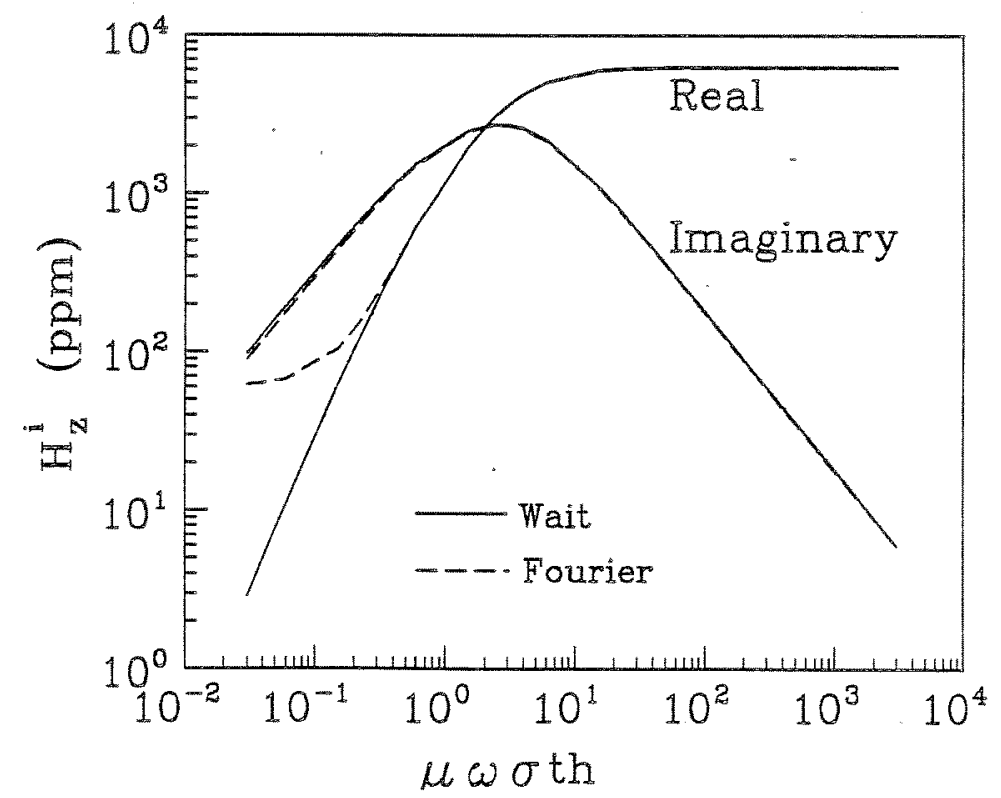

EIGURE 3.2. The response curve (in parts per million of the exciting field at the receiver) for a vertical transmitter and recelver dipoles 30 metres high separated by 9 metres. The 'Wait' solution - solid line, Eourler domain solution dashed line, the grid for the Eourier domain case is 128 nodes by 128 nodes with 4 metres separating nodes. 
Dipoles oscillating at different frequencies induce different current systems in the sheet. The currents induced at low frequency will be of a large lateral extent, and near the source the imaginary (quadrature) component will dominate. At high frequencies the current systems will be smaller, and dominated by the real (in phase) component.

The frequency response for a sheet of constant resistance $(\mathrm{R}=0.01$ Ohms) is shown on figure 3.2 . The receiving dipole is 9 metres from the transmitting dipole, and both dipoles are $30 \mathrm{~m}$ above the sheet. Here the 'Wait' solution is designated by the solid line, and the Eourier domain solution by the dashed Iine. The grid used for the Eourier domain case has 128 by 128 nodes with a nodal spacing of 4 metres. At different frequencies the induced current systems are different sizes. The sampling density of the magnetic field must therefore vary according to the size of the induced currents. At low Irequencies the imaginary component of the induced field will be of approximately the same lateral extent as the exciting field. The real component will have a larger lateral extent, and thus the resulting real field will be inadequately sampled--hence the errors at low response parameter ( $\mu \omega \sigma$ th).

A compromise must be made between sampling out to infinity. sampling the exciting fields optimally, sampling the induced (secondary) fields optimally, and describing the resistance variation optimally.

Eigure 3.3 shows the the result of varying the sampling interval (or grid spacing). This is a plot of the percent error in the field at the receiver calculated using the Eourier domain 


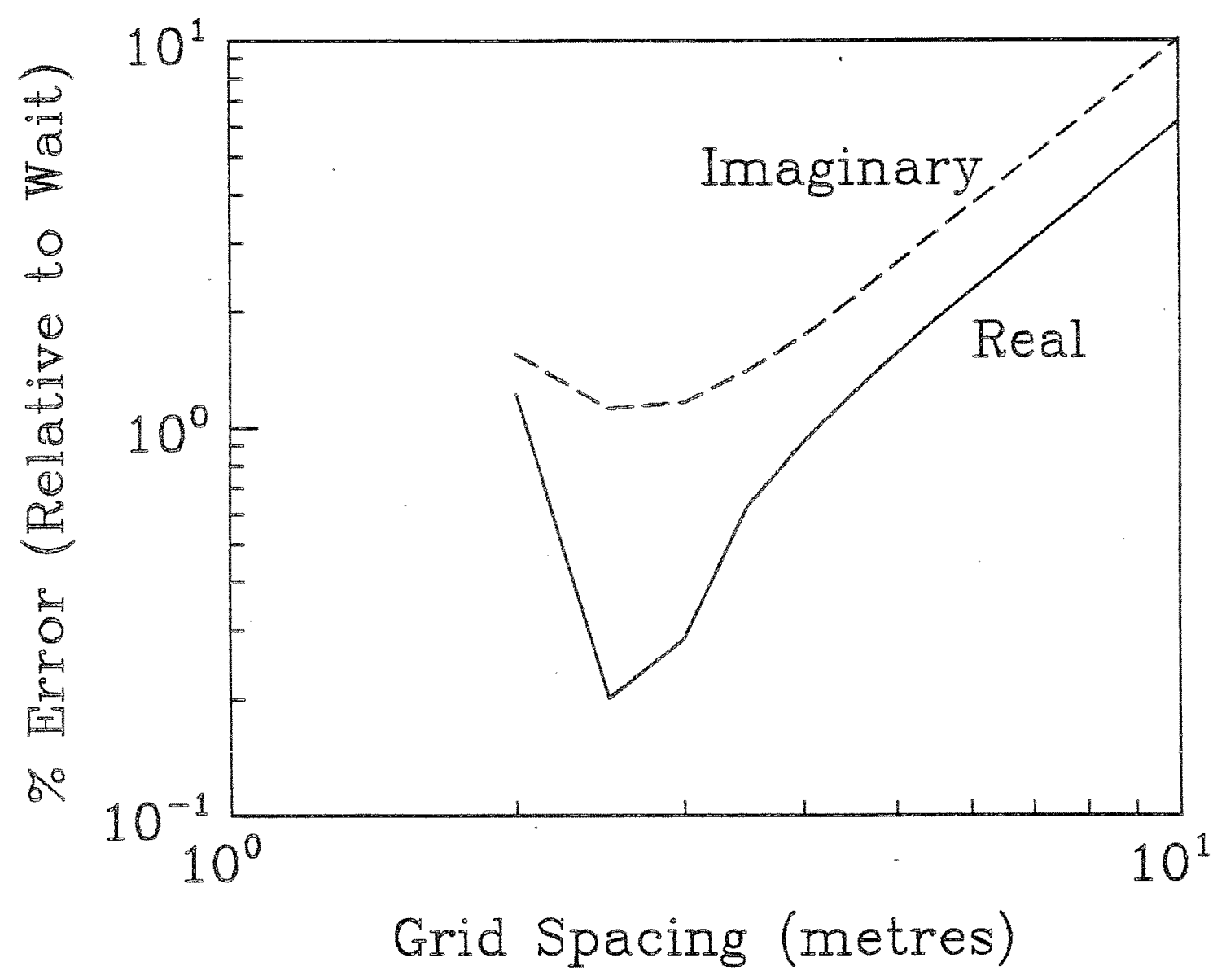

EIGURE 3.3 The percent difference between $H_{z}^{i}$ at the receiver as calculated by the Fourier method compared to Wait's method plotted as a function of the grid sampling interval for the Eourier domain grid. The flight height = 30m, $\mu w O t=.2 \mathrm{~m}^{-1}$, and the dipole separation $=9 \mathrm{~m}$. 
solution when compared against the 'Wait' solution. For this graph the parameters are: a flight height of $30 \mathrm{~m}$, a transmitter/receiver separation of 9 metres, and $\mu w \sigma t=.2$ inverse metres. The large errors at small grid spacing are due to the multiple sources outside the sampled grid. The error at large grid spacing is due to aliasing as the data has been sampled too sparsely. In general the optimal spacing for the imaginary component is different from that of the real component. Similar plots were generated for many different combinations of $\mu w o t$. In all cases the transmitting and receiving dipoles were vertical and separated by 9 metres. The grid used was 128 nodes by 128 nodes.

The optimal spacing has been found empirically for five dipole heights and these have been plotted as a function of 'response parameter' ( $\mu \omega \sigma t h)$ on figures 3.4 to 3.6. The optimal spacing decreases with increasing response parameter. On figure 3.6 the graph shows resistive (low response parameter) and inductive (high response parameter) limits.

Eor practical reasons the matrix $\{A+C I\}$ can only be inverted for a limited number of wavenumbers, and thus the Fourier transform of the induced field is truncated. A series of experiments was done to filter the induced field so that the wavenumber components fall off smoothly--tending towards zero at the truncation distance in $(p, q)$ space. This was done for an exciting frequency of $500 \mathrm{~Hz}$, a dipole separation of 9 metres, a nodal separation of 4 metres, a sheet with $R=0.01$ Ohms, and a transmitter/receiver height of 30m. Kanasewich (1981) shows that a truncation of the Eourier transform of a field can result in negative side lobes when back-transformed to the space 
Real and Imaginary; dipole height shown

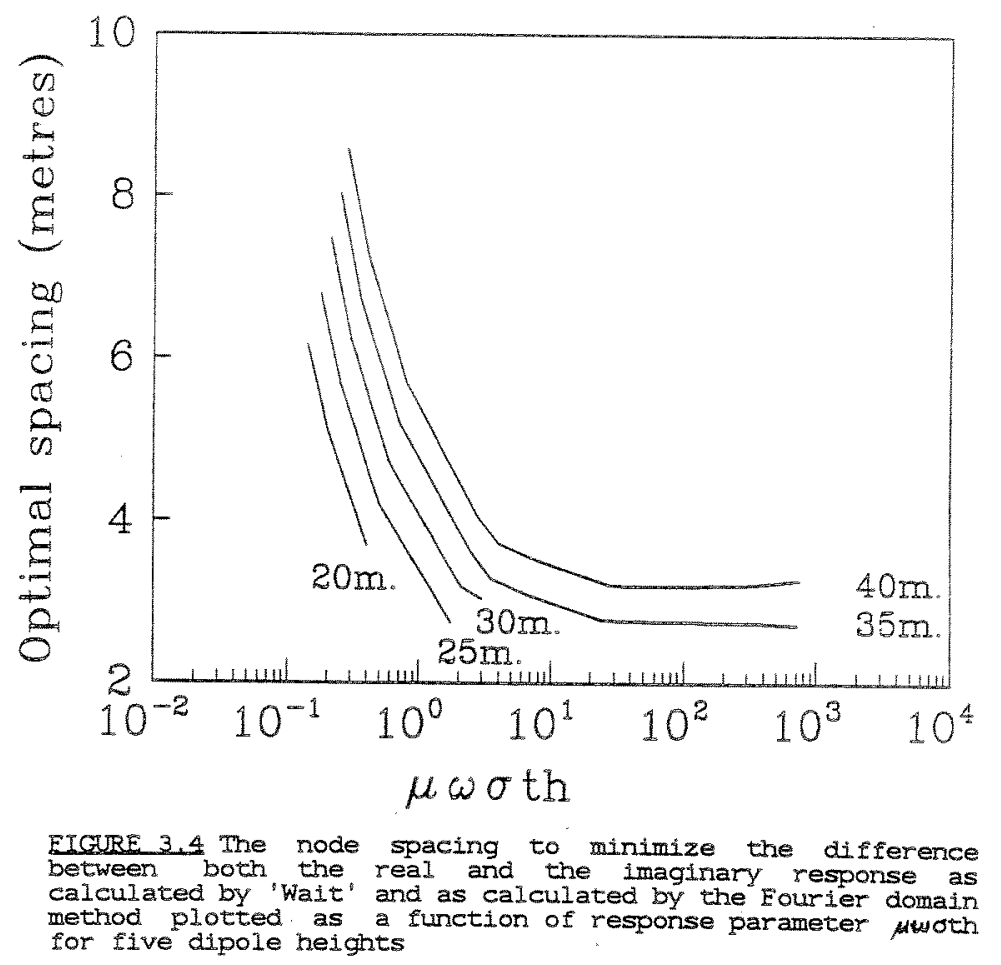

Real component; dipole height shown

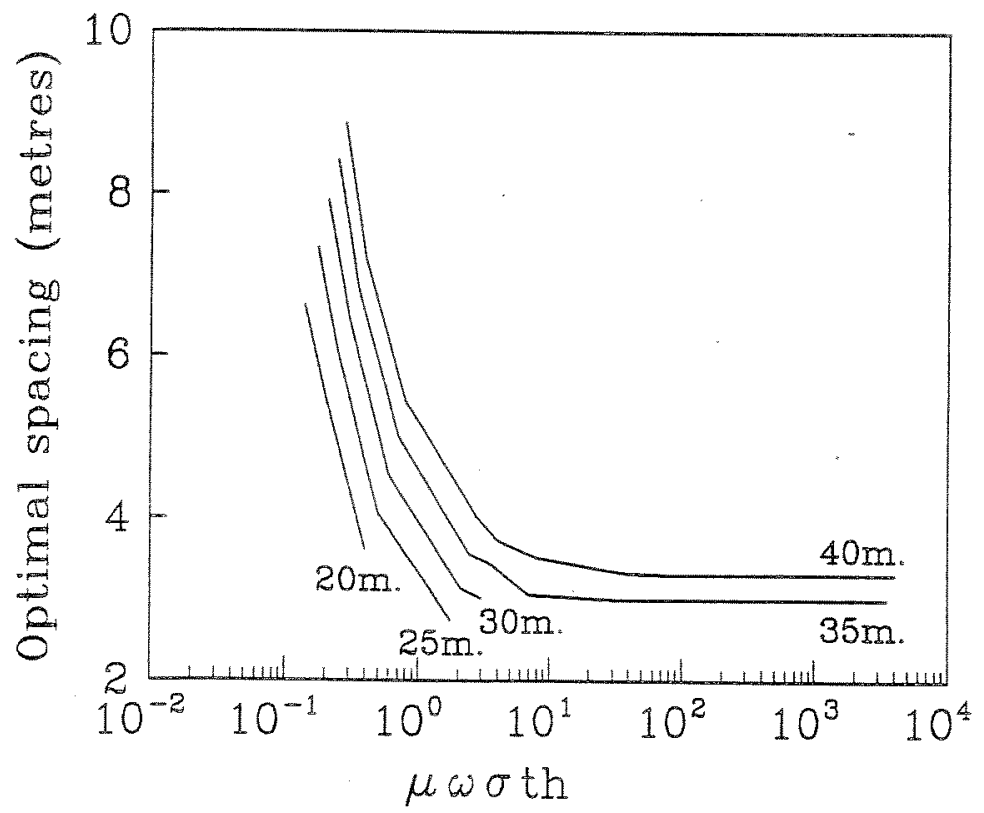

FIGURE 3.5 The node spacing to minimize the difference between the real response as calculated by 'Wait' and as calculated by the Eourier domain method plotted as a function of response parameter $\mu$ woth for five dipole heigints 


\section{Imaginary ; dipole height shown}

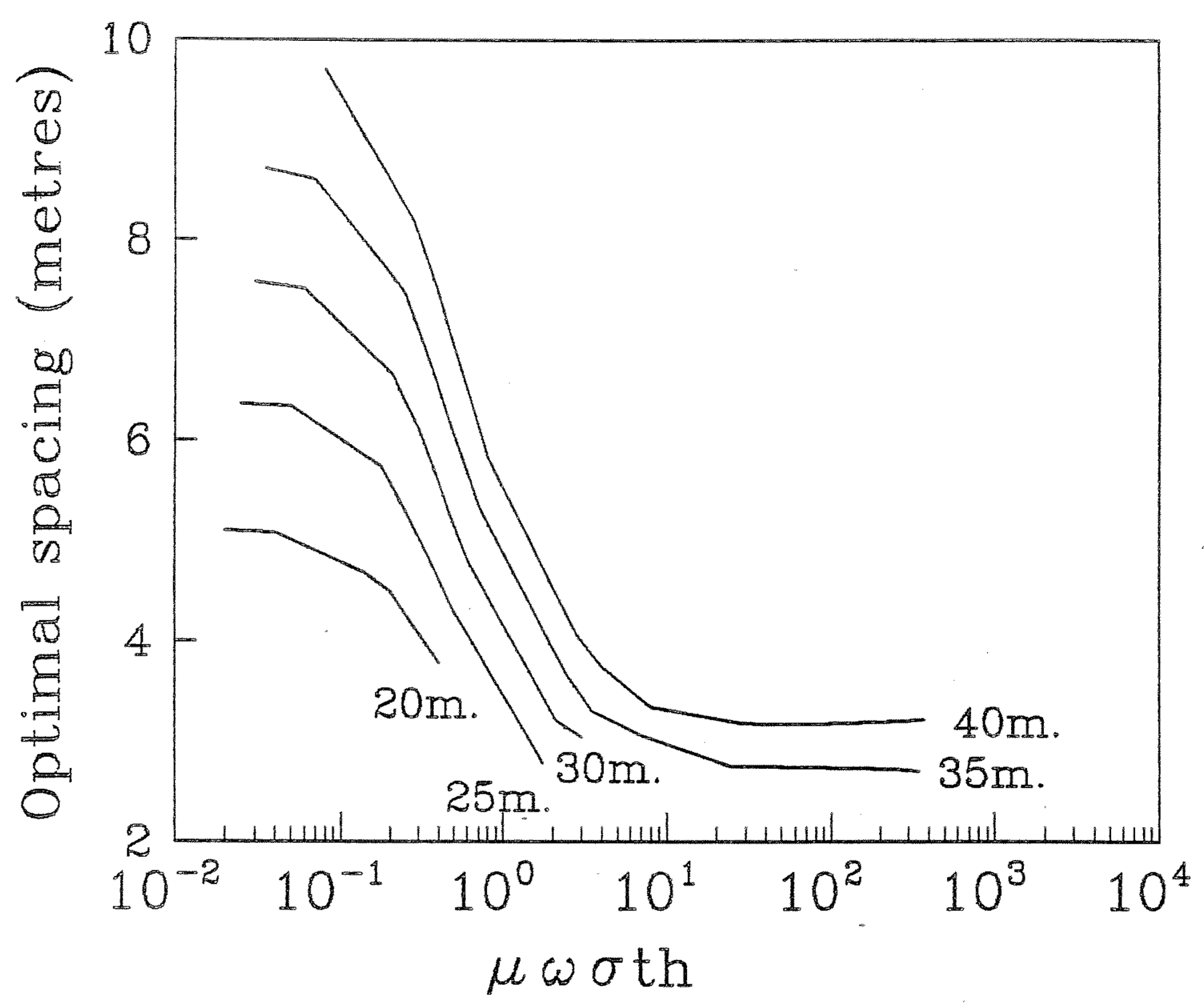

EIGURE 3.6 The node spacing to minimize the difference between the imaginary response as calculated by 'Wait' and as calculated by the Eourier domain method plotted as a function of response parameter uwoth for five dipole heights 
domain. The Parzen and the Bartlett filters (Kanasewich 1981) will not give negative side lobes in the space domain when they are applied to the Fourier transform in the wavenumber domain. These filters have been applied to the Eourier transform of the induced field so as to leave the number of wavenumbers shown on figure 3.7. The solid line is the vertical field at the receiver obtained using 16284 wavenumbers. The computer time required to invert for the number of wavenumbers shown in figure 3.7 increases rapidly as the number of wavenumbers increases (see figure 3.8). Using the method of solution which converges most rapidly will save computation time. For this purpose simple truncation is best.

The Eourier method described is therefore capable of calculating the response of a uniform sheet when excited by the field of an arbitrarily shaped exciting source (provided that the field of that source can be calculated and sampled sufficiently well). The source field, the resistance and the induced field must however be adequately sampled. The work presented in this chapter has only-been for a vertical transmitting dipole and a 128 node by 128 node grid. Thus a similar empirical excerise must be undertaken for other source fields and grid dimensions

For different frequencies and resistance distributions a different discretization grid must be used. This makes the calculations labourious, hence conversion of the results to the time domain would be very difficult. 

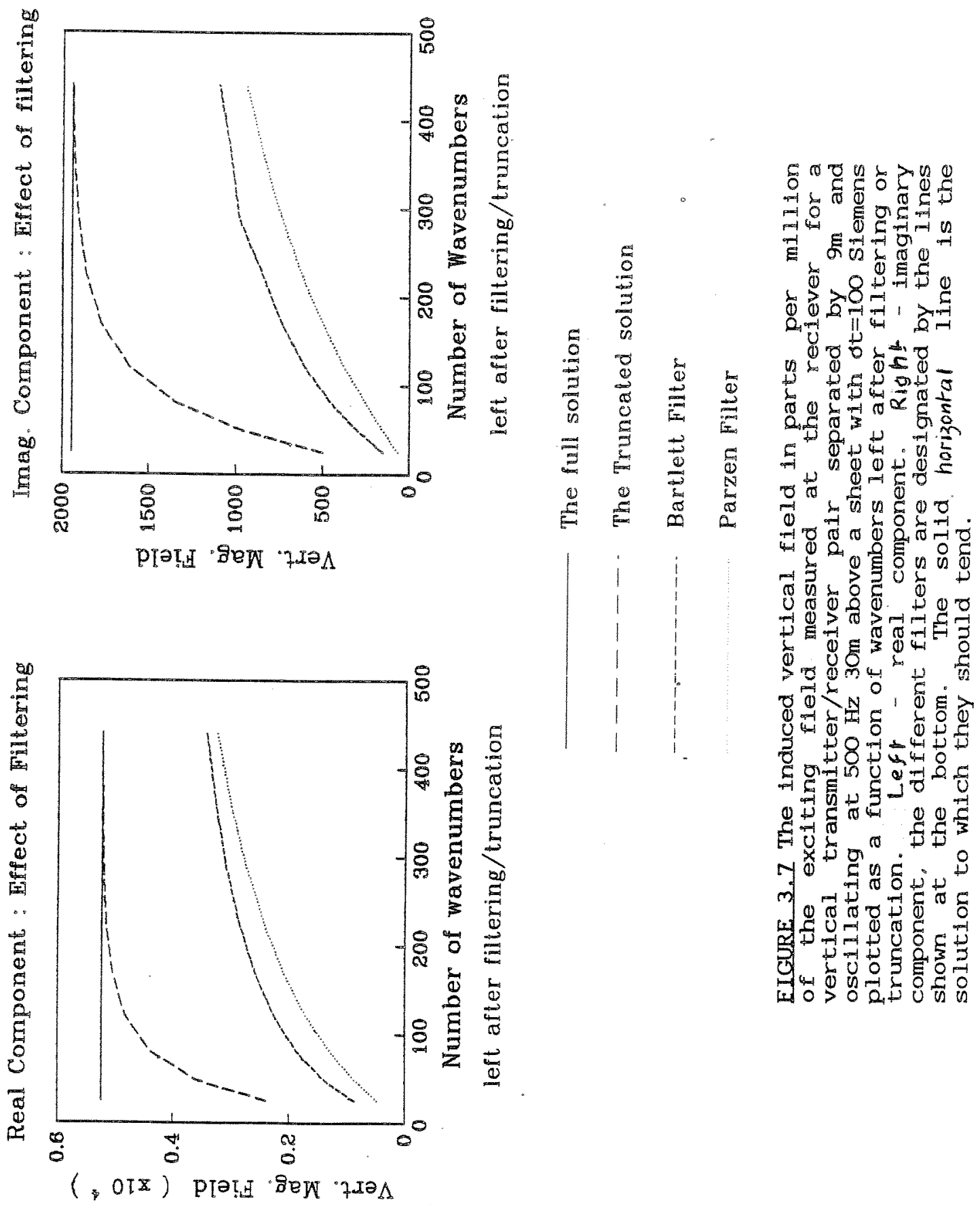


\section{Computing Time}

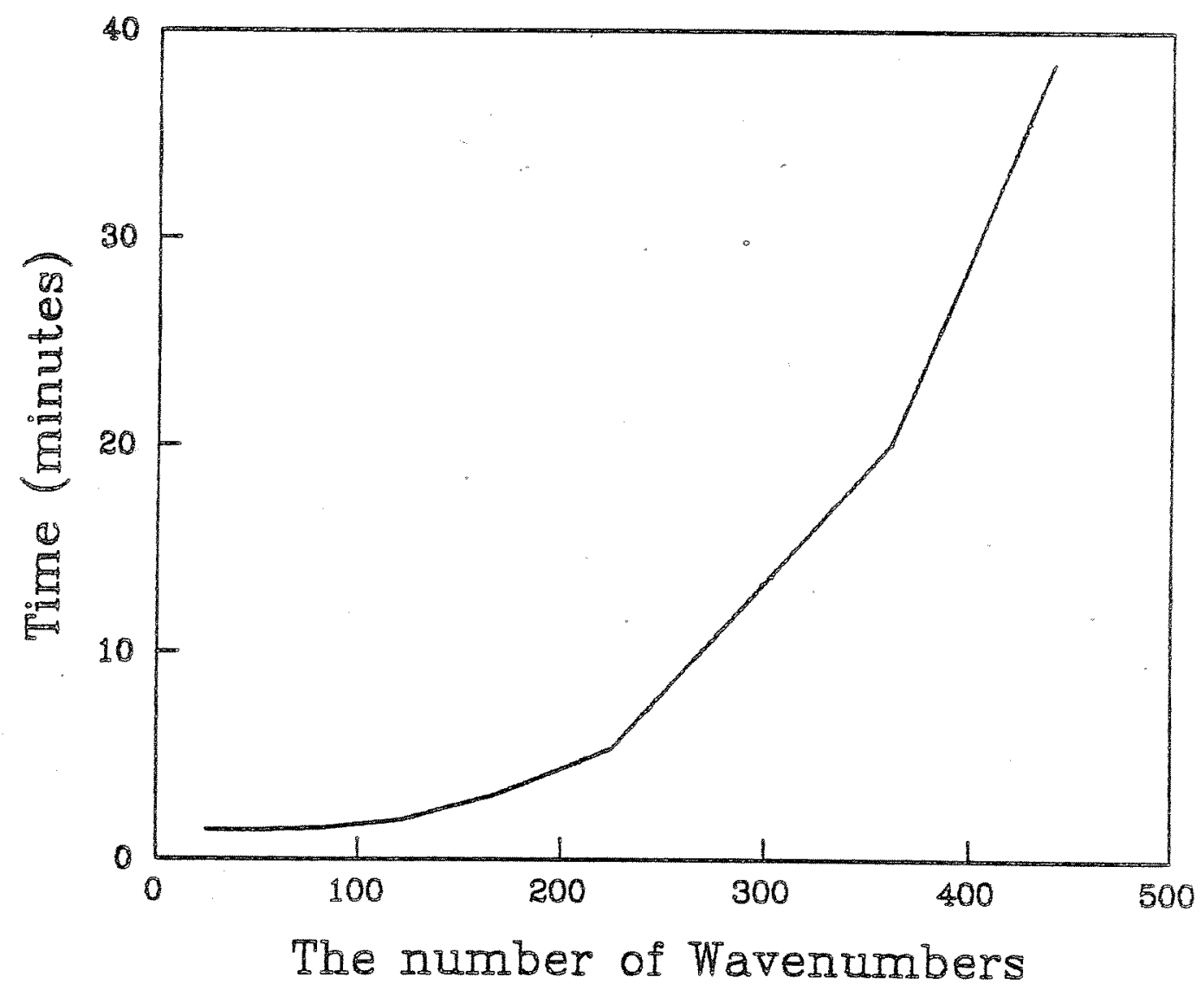

EIGURE 3.8 The computation time taken on a VAX 11/750 to invert a complex matrix (using a method due to Groom - pers com (1984)) as a function of the number of variables (wavenumbers) solved for. 


\subsection{The Variable Conductivity Case}

Type curves have been computed for the conductivity distribution shown on figure 3.9. The conductivity contrast is varied so that the contour shapes remained the same, but the contrasts change from 100:10 Siemens to 100:31, 100:920 and 100:9100 Siemens. The surrounding conductivity thickness product (ot) is always 100 siemens. The type curves shown in figure 3.10 are for a helicopter EM system with a vertical dipole transmitter and a vertical dipole receiver separated by 9 metres. The dipoles are 40 metres above the ground, and the operating frequency of the system is $300 \mathrm{~Hz}$ (this is quite close to the inductive limit). The response has been plotted in parts per milition (ppm). The grid size (chosen from figure 3.4) is 4.0 metres.

Away from the anomalous area of conductivity the response tends to the uniform sheet response--which is as expected. The conductive cases appear to tend towards the response expected for a inhomogeneity which is infinitely conductive. In such a case the real response should increase to the inductive limit, and the imaginary response should decrease to zero. For these curves the wavenumber components near the truncation distance are almost negligible and thus the effect of the wavenumbers outside the truncation distance will be minimal. Ignoring the wavenumbers outside the truncation distance will therefore not result in significant errors.

Eor the resistive inhomogeneity the real component of the response is expected to decrease as the conductivity decreases (as is shown), and the imaginary component is expected to 


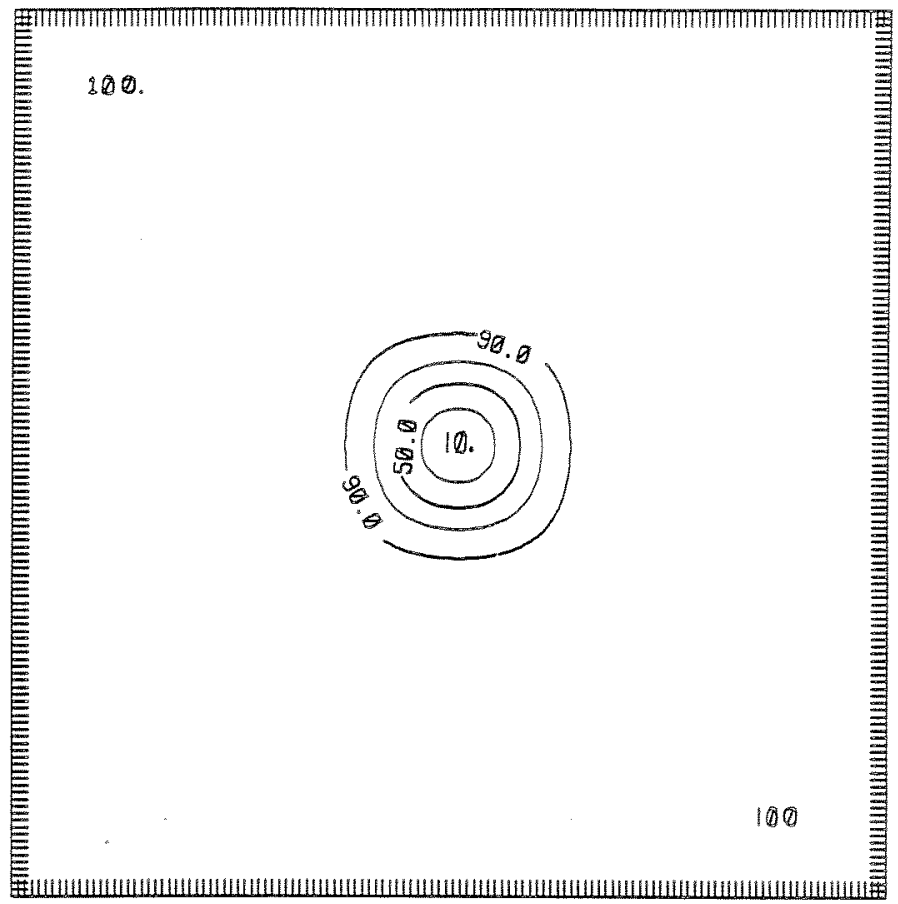
(plotted in Seimens). The full grid of discretisation is shown (from -256m to 252m in the $x$ and $y$ directions - the node spacing is 4 metres) The surrounding conductivity thickness product is 100 Siemens, but in the centre this decreases to 10 Siemens.

Flight Height $40 \mathrm{~m} ; 300 \mathrm{~Hz}$

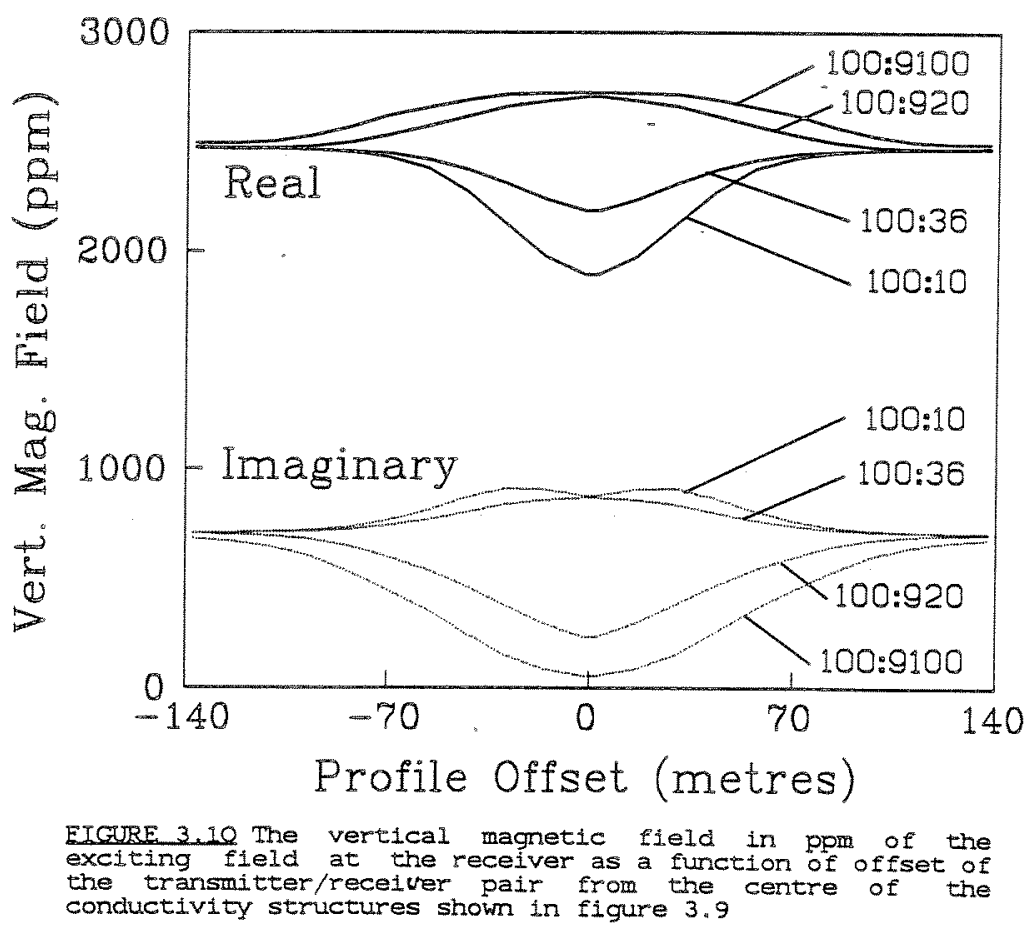


increase. The two peaked form of the imaginary response occurs because when the dipole pair is over the edge of the "resistive hole" less current will be induced than when the dipole pair is over the centre. This is because the currents must be induced on the far side of the hole--away from the edge that the dipole pair is over. This area is a long way from the dipole, and the currents induced will be weak and hence the response will be characteristic of a more resistive sheet. When the dipole is in the centre of the hole, the currents can be induced in the conductive regions outside the hole with less difficulty, as the conductive areas are not so far from the dipole. Therefore the shape of the profiles makes intuitive sense. Even so, it would be best to check these type curves against another numerical model or a scale model before making further comments on the accuracy of the solution.

\section{2 .1 Numerical Checks}

Program "PLATE" (Dyck et al (1980)) was used to generate vertical dipole-dipole Helicopter EM data in profile form. The model used is a flat lying horizontal plate 140 metres by 140 metres. This is within the bounds of accuracy of program PLATE. The source is a dipole 40 metres high oscillating at $1000 \mathrm{~Hz}$. The vertical transmitter and receiver dipoles were separated by 9 metres. The conductivity thickness product of the plate is 100 Siemens. A profile of the conductivity used by the PLATE program, and that used by the Eourier domain method are shown on figure 3.11. The results are shown on figure 3.12 . 
Page 20a

Height $40 \mathrm{~m} ; 1000 \mathrm{~Hz}$; Plate $140 \mathrm{~m} \times 140 \mathrm{~m}$

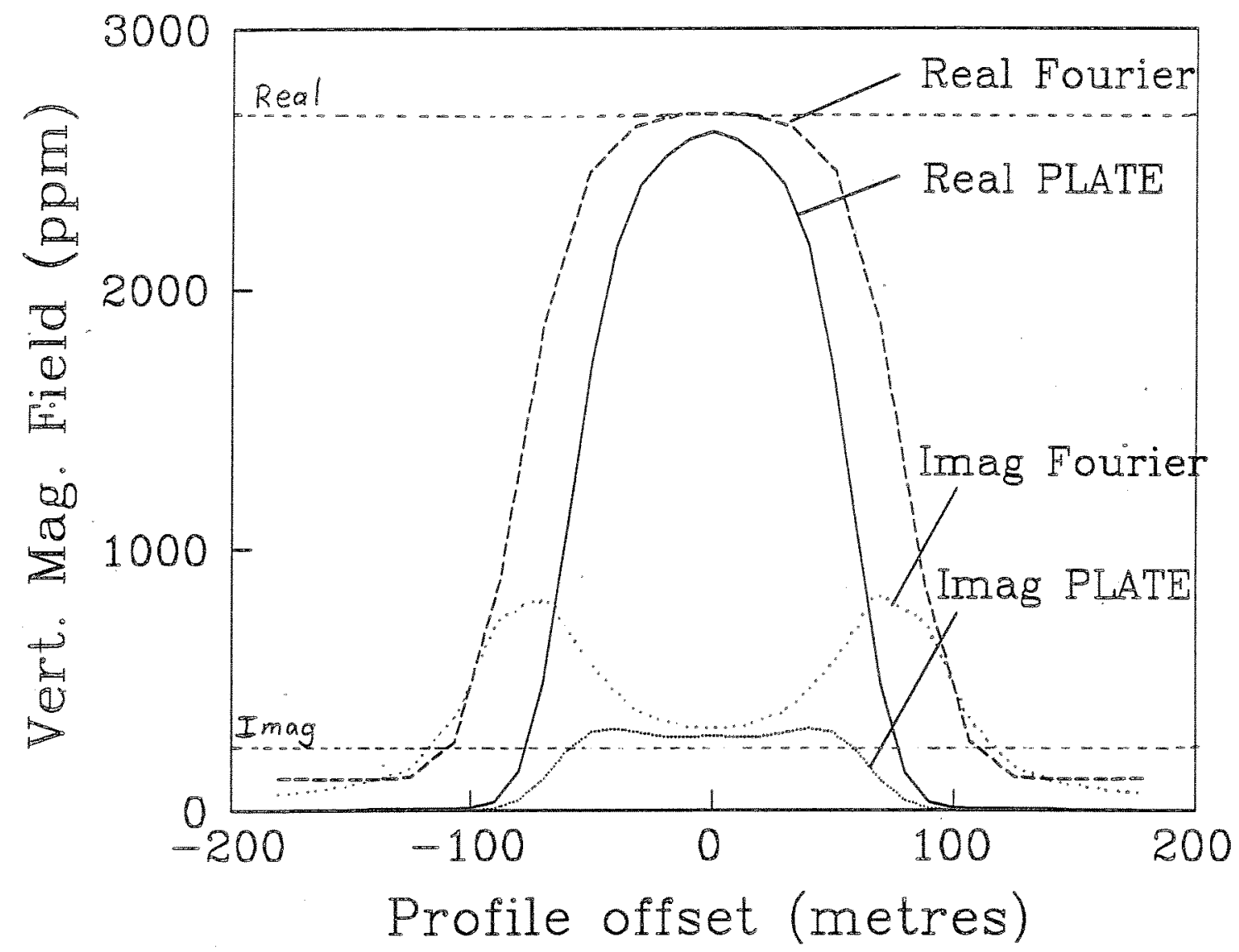

EICURE 3.12 The response (in ppm) as calculated by PLATE and the Fourier domain method for the respective conductivity distributions shown in figure 3.11 plotted as a function of the offset of the transmitter/receiver coil pair (which are separated by 9 metres and 40 metres vertically above the sheets). The exciting frequency is $1000 \mathrm{~Hz}$. The dashed horizontal lines are the real (top) and imaginary (bottom) response for a uniform thin sheet with ot $=100$ siemens.

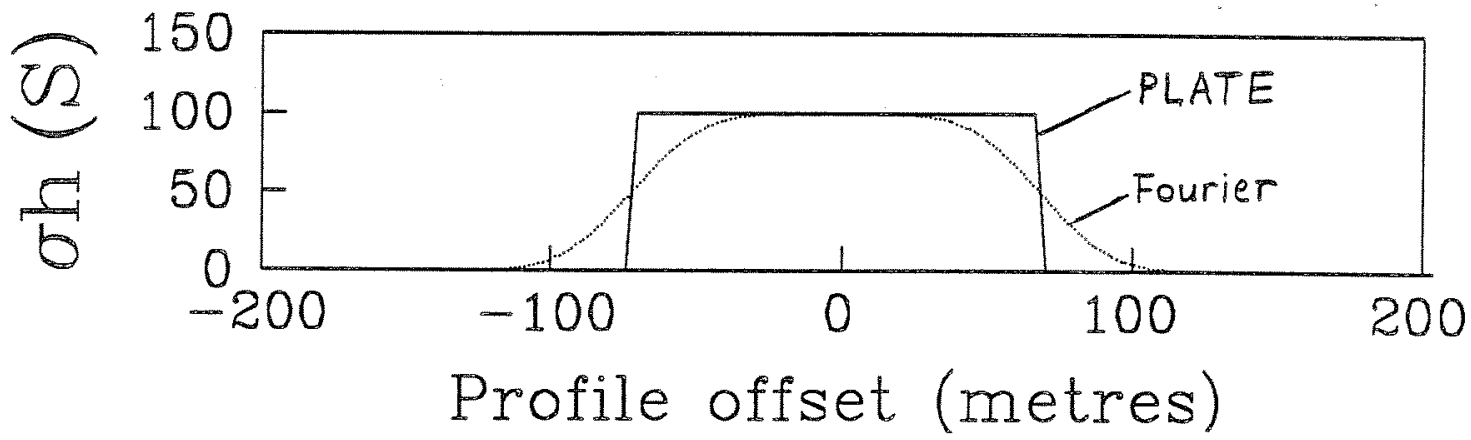

EICURE 3.11 A profile along the $x$ axis of the distribution of the conductivity thickmess product (Siemens). The solid curve is the distribution used by program PIATE, and the dotted curve that used by the Eourier domain method. The profile along the $Y$ axis is identical. 
The Eourier method solved for 361 wavenumbers (a 20 minute task on the VAX 11/750), and represented $R(x, y)$ with approximately 12000 wavenumbers in the $(p, q)$ domain. The dashed horizontal lines across figure 3.12 are the responses for the uniform thin sheet (ot=loos) calculated by the Eourier domain method. As expected the results from both methods tend towards the uniform thin sheet values near the centre of the conductive area. The conductivity distribution shown in figure 3.11 is wider for the Eourier method than the PIATE method. It is therefore no surprise that the anomaly pattern is wider and larger, as the Fourier domain induced fields would not 'see' the edges of the inhomogeneity as they would in the PLATE case. For the Eourier domain method the imaginary response is larger close to the edges of the plate.

If not enough wavenumbers were solved for initially, then increasing the number of wavenumbers used to represent the induced field should change the response obtained. When 441 wavenumbers were solved for similar results to those in figure 3.12 were obtained, and this suggests that the larger imaginary response near the edges is a real physical effect.

Although the results obtained are different. the conductivity structure is also different. The fact that the results tend to the same limit, and that the Eourier domain method has converged when the wavenumbers solved for increases suggests that both methods are correct. The Fourier method is therefore capable of modelling the response for the conductivity anomaly shown in figure 3.11 for the system parameters described above. 


\subsection{CHAPTER 4 - TIME DOMAIN SOLUTION}

From equation 2.0 .17 in Chapter 2 the governing differential equation is:

$$
R(x, y) \operatorname{div}\left(H_{q}^{i}\right)+\operatorname{grad}(R) \cdot\left(H_{i}^{i}\right)=-\frac{\mu}{2} d H_{Z} / d t
$$

where div and grad are two dimensional operators in the $z=0$ plane. This simplifies to:

$$
-\mathrm{R}(\mathrm{x}, \mathrm{y}) \frac{\partial \mathrm{H}_{z}^{i}}{\partial z}+\frac{\partial \mathrm{R}}{\partial \mathrm{x}} \mathrm{H}_{x}^{i}+\frac{\partial \mathrm{R}}{\partial \mathrm{y}} \mathrm{H}_{y}^{i}=-\frac{\mu}{2} \frac{\partial \mathrm{H}_{z}}{\partial t} .
$$

Erom 2.2.0, and 2.2 .1 at $z=0+$

$$
\begin{aligned}
& H_{z}(x, y) \ll-\infty-i p H_{2}(p, q) /\left(p^{2}+q^{2}\right)^{1 / z} \\
& H_{y}(x, y)<-\infty-i q \vec{H}_{z}(p, q) /\left(p^{2}+q^{2}\right)^{1 / 2}
\end{aligned}
$$

and from 2.2 .4

$$
-\frac{\partial H_{2}}{\partial z}<\cdots\left(p^{2}+q^{2}\right) \bar{H}_{z}(p, q) \text {. }
$$

The transmitter of the E.M. system under consideration carries a constant current $i(t)=I$ which is switched off instantaneously. The constant current has a magnetic field $\mathrm{H}$ associated with it.

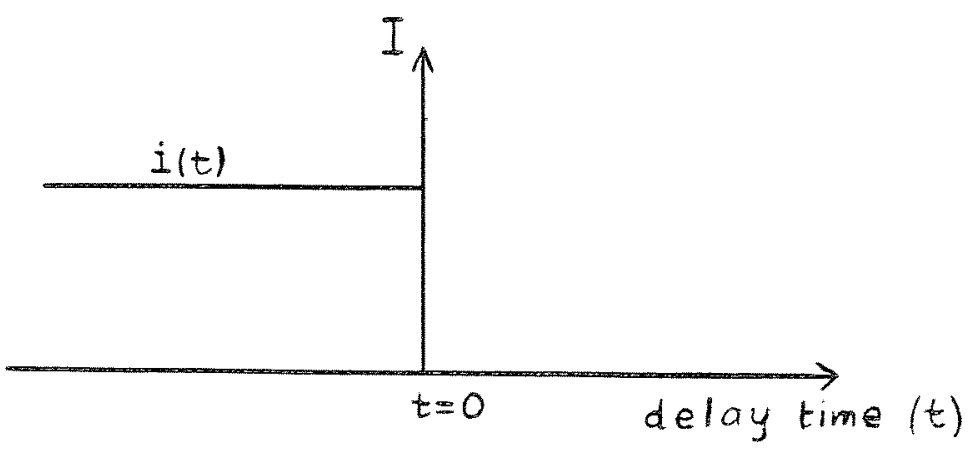


When the primary (exciting) current is switched off a secondary (induced) current is induced in the plate, so as to conserve the magnetic field everywhere prior to switch off. This induced current then decays in a manner described by equation 4.0 .1 .

The initial induced field $\mathrm{H}_{z}^{i}$ at $t=0$ is thus equal to the exciting field $H_{z}^{e}$. The left hand side of 4.0 .1 can be calculated, and the normal component of $H$ can be stepped through time using a finite difference formula.

\subsection{The constant conductivity Case.}

As in Chapter 3 (where the frequency domain case was considered) insight into the time domain problem can be gained by looking at the constant conductivity case. If the conductivity is constant, then $d R / d x=0$ and $d R / d y=0$. Thus from 4.0 .1

$$
\mathrm{R} \frac{\partial H_{z}^{i}}{\partial z}=-\frac{\mu}{2} \frac{\partial H_{z}}{\partial t}
$$

If the exciting field measured on the sheet is given by $H(x, y, z)$ at $t=0$ seconds (where $z$ is the distance of the source from the sheet), then the solution at delay time $t$ is $H(x, y, z \pm 2 t R / \mu)$. The + sign applies when $z$ is positive, and the - sign when $z$ is negative. This is known as the Maxwell retreating image solution (Grant and West (1965)). It is described as the retreating image solution because the field at delay time $t$ is the field which would be associated with an image of the source that moves away from the sheet with a velocity equal to $2 R / \mu$. At $t=0$ the image is the same distance from the sheet as the 
source, but on the opposite side of the sheet.

Taking the Eourier transform of 4.1 .0 gives

$-R\left(p^{2}+q^{2}\right)^{1 / 2} \vec{H}_{2}^{i}(p, q)=\frac{\mu}{2} \frac{\partial}{\partial t}\left(\vec{H}_{2}(p, q)\right)$

so.

$$
-(2 R / \mu)\left(p^{2}+q^{2}\right)^{1 / 2} \bar{H}_{2}^{i}(p, q)=\frac{\partial}{\partial t}\left(\bar{H}_{2}(p, q)\right) .
$$

This is an ordinary differential equation with time as the independent variable. The solution to this differential equation is:

$$
\mathrm{H}_{2}(p, q, t)=\mathrm{H}_{2}(p, q, 0) \exp \left(-(2 \mathrm{R} / \mu)\left(p^{2}+q^{2}\right)^{1 / 2} t\right)
$$

When calculating the discrete Eourier transform aliasing problems can be avoided by checking that the discrete Eourier transform of the initial field tends to zero as the wavenumber components approach the Nyquist iimit.

Eor the uniform (constant conductivity) thin sheet, the field will decay as a function of delay time, the wavenumber components being attenuated according to 4.1 .1 . The solution should not be taken too far in time, as eventually only very low wavenumber components will contain information. Otherwise stated, the sampling of the fields becomes too dense but not extensive enough. Consider the circle in wavenumber domain which has a radius of half the Nyquist (ie $\lambda_{N} / 2$ if the Nyquist wavenumber $\left.\lambda_{N}=\left(p_{N}^{2}+q_{N}^{2}\right)^{1 / 2}\right)$. A reasonable scheme would be to time step the fields until the magnitude of all the Eourier components with wavenumbers outside this circle first become 
negligible. The grid size could then be doubled with negligible aliasing occurring and the fields would again be represented in an optimal manner. This process of grid expansion can be repeated, until the fields have been obtained at the latest delay time required.

An indication of the delay time that grid expansions will be required can be obtained by analyzing the uniform thin sheet response. Thus it is possible to select an appropriate sized time step.

Require that at delay time $t=t^{\prime}$ the magnitude of the wavenumber components near the Nyquist be less than 1/a times the magnitude of the wavenumber components near the first harmonic (typically 'a' may be 100.). Thus

$$
\left|\overline{\mathrm{H}}_{2}\left(\lambda_{N}, t=t^{\prime}\right)\right|<1 / \mathrm{a}\left|\overline{\mathrm{H}}_{2}\left(p_{1}, q_{1}, t=t^{i}\right)\right|
$$

where $p$, and $q$, represent the first wavenumber components in the $p$ and $q$ directions. The time steps should continue until delay time $t^{\prime \prime}$ when

$$
\left|\vec{H}_{2}\left(\lambda_{N} / 2, t=t^{\prime \prime}\right)\right|<1 / a\left|\vec{H}_{2}\left(p_{1}, q_{1}, t=t^{\prime \prime}\right)\right|:
$$

At this point the grid size can be doubled, without a significant amount of information being lost due to folding back of wavenumbers around the Nyquist.

If at delay time $t=t^{\prime}$ the ratio

$$
\overline{\mathrm{H}}_{2}\left(\lambda_{w} / 2, t=t^{\prime}\right)|/| \overrightarrow{\mathrm{H}}_{2}\left(p_{1}, q_{1}, t=t^{\prime}\right) \mid=1 / \mathrm{b} .
$$


then using $4.1 .1,4.1 .2$ and 4.1 .3 gives

$$
t^{\prime \prime}-t^{\prime}=\frac{\ln (a / 0)}{v\left(\left(p_{N}^{2}+q_{M}^{2}\right)^{1 / 2} / 2-\left(p_{1}^{2}+q_{1}^{2}\right)^{1 / 2}\right)}
$$

where

$$
v=(2 R / \mu)
$$

In practice the process of ensuring that the magnitudes of the wavenumber components have decayed away is done in a fairly crude manner. Consider the case of a 128 by 128 grid. On the $p$ axis eight wavenumber components on either side of the zero wavenumber component are examined. The one with the largest amplitude is chosen to represent the wavenumbers near the first harmonic (call this $p_{f}$ ). The spectral amplitude which represents components near the half Nyquist wavenumber (call this $p_{h}$ ) is found by finding the maximum amplitude for eight wavenumbers either side of the half Nyquist. The amplitude at the Nyquist is taken to be the average of the amplitudes of the three wavenumbers below the Nyquist and the Nyquist itself. These quantities are also obtained for the $q$ axis (defining $q_{\text {f }}$ and $\left.q_{h}\right)$. The values of $\left(p_{N}^{2}+q_{N}^{2}\right)^{1 / 2} / 2$ and $\left(p_{1}^{2}+q_{1}^{2}\right)^{1 / 2}$ in 4.1 .5 are modified acordingly, the values of $a$ and $b$ (in (4.1.3) and (4.1.4)) are selected, and the time between grid expansions can be calculated from 4.1 .5 .

The most efficient program for calculating the decay of the fields will take time steps such that the wavenumber components will decay in the minimum number of time steps possible. From 4.1 .1 it can be seen that the wavenumber components decay exponentially. 'Thus if the steps sizes are equal, then the 
initial step will account for a large percentage of the decay. The time stepping process actually involves an addition and not a multiplication (as may be suggested by equation 4.1.1), and thus care must be taken in choosing the step size. For example if a time step is taken which decreases the magnitude of wavenumbers at half the Nyquist by fifty percent then the wavenumber components near the Nyquist (which are expected to decrease more) may have such a large an amount subtracted that it results in changing their sign. This will cause erroneous answers .

One solution to the above problem would be to make the initial step size smaller. This will improve the accuracy of the answer, but it may result in the time stepping process taking too long (because the amount the fields are decreased by at each step decays exponentially). The step size could be increased at each step by a multiplicative factor. This will make the computation more efficient. It also makes some intuitive sense, as the change in the fields per unit delay time is less rapid at later delay times, and thus a greater step size can be used.

Eor equal step sizes, the step size (h) can be found as follows

$$
h=\left(t^{\prime \prime}-t^{\prime}\right) / N
$$

where $N$ is the number of steps required between grid expansions. Eor increasing step sizes the first step can be calculated using the following formula: 


$$
h=\left(t^{\prime \prime}-t^{\prime}\right) / \sum_{j=0}^{N} s^{j}
$$

where $s$ is the time step expansion factor, and the kth step size is given by

$$
h_{k}=\operatorname{sh}_{k-1} \text {. }
$$

If the step size becomes too large prior to a grid expansion, then noise can be generated. Figure 4.1 shows a profile of the magnetic field decaying. Each curve represents the fields at a different delay time. The initial field (the largest amplitude) is the field associated with a 40 by 40 metre loop 10 metres above a sheet with a conductivity thickness product equal to 100 siemens. The oscillatory field is at the latest delay time. Eigure 4.2 is a plot of the wavenumber components along the $p$ axis at delay time $t=0$. Eigure 4.3 shows the wavenumber components at the latest delay time $(t=1.4$ milisec). Initially 43 wavenumbers along the p axis are used to describe the field. At the later time (after 15 time steps) the field can be represented with 11 wavenumbers along the $p$ axis, and most components in the transform are devoid of information. Note that the high wavenumber components now contain noise. This noise is probably a result of subtracting an amount from the field which contained more high wavenumber information than the field contained itself. Once noise like this develops it grows very rapidly.

As the decay of the wavenumber components is known from 4.1.1. it is possible to predict when a wavenumber component should have decayed away to a negligible value. A filter could then be applied so as to assure that this negligible value remains negligible. At each time step the filter will change as 
Without expanding the grid size

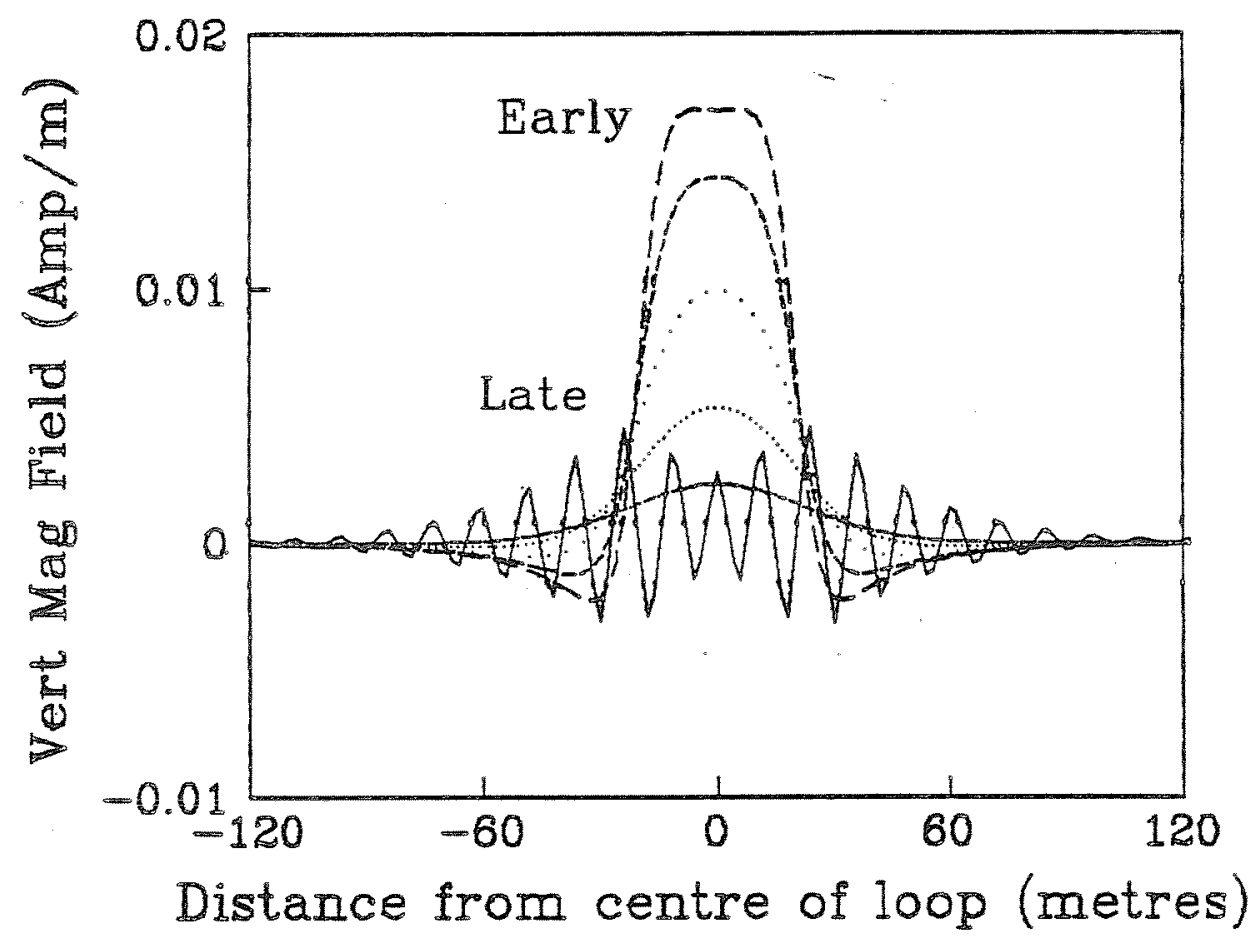

EIGURE 4. The vertical magnetic field (Amo/m) at six different times. The largest amplitude response (dashed) is at the earliest time. The dotted lines are the field later. whilst the solid (oscillatory line) is at the latest time. The exciting loop is $40 \times 40 \mathrm{~m}$ 10m above the sheet. $0 t=100 \mathrm{~s}$. The discretization grid has nodes from $-128 \mathrm{~m}$ to $126 \mathrm{~m}$ (in the $x$ and $y$ direction) separated by $2 \mathrm{~m}$. 


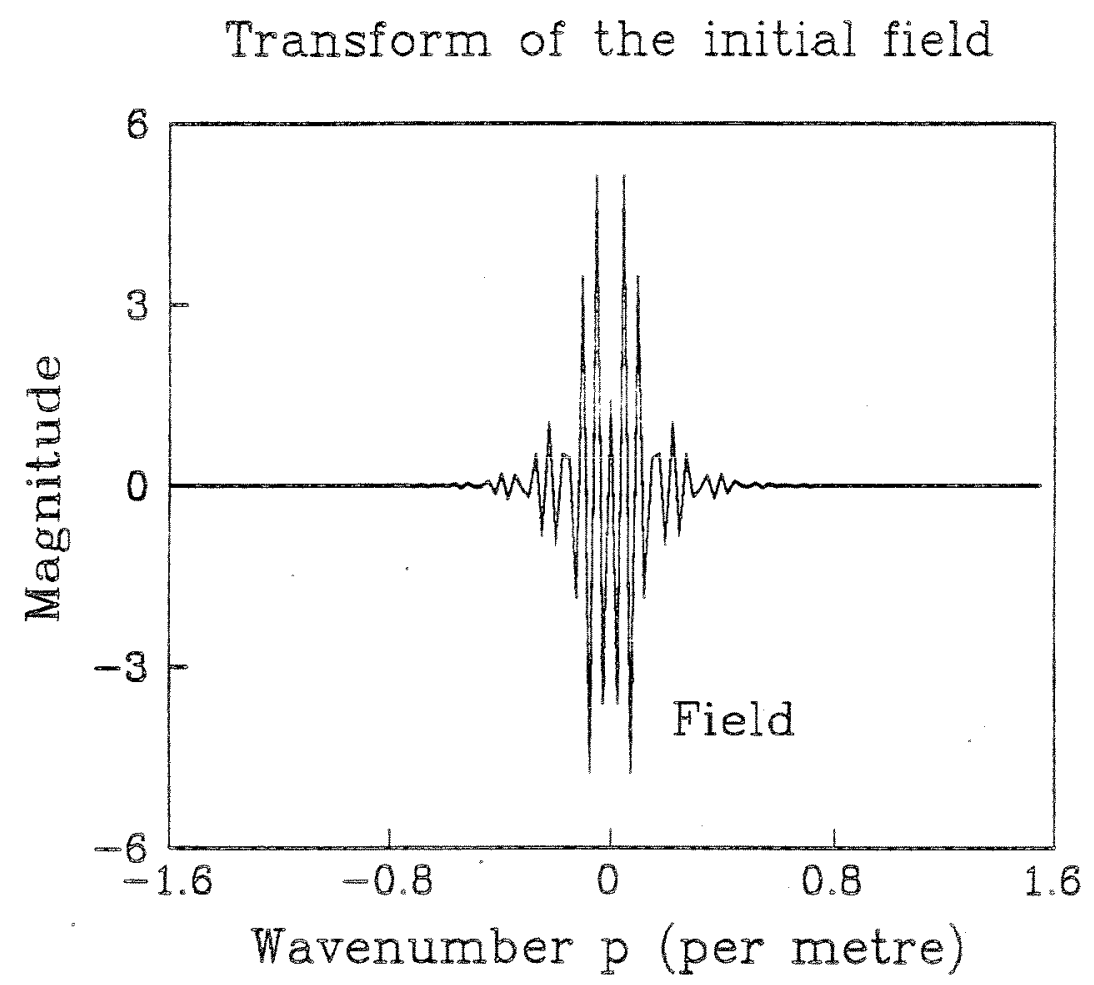

EICURE 4 . The $p$ axis values for the discrete Eourier transform of the initial Eleld (largest ampiltude) in Eigure 4.1 .

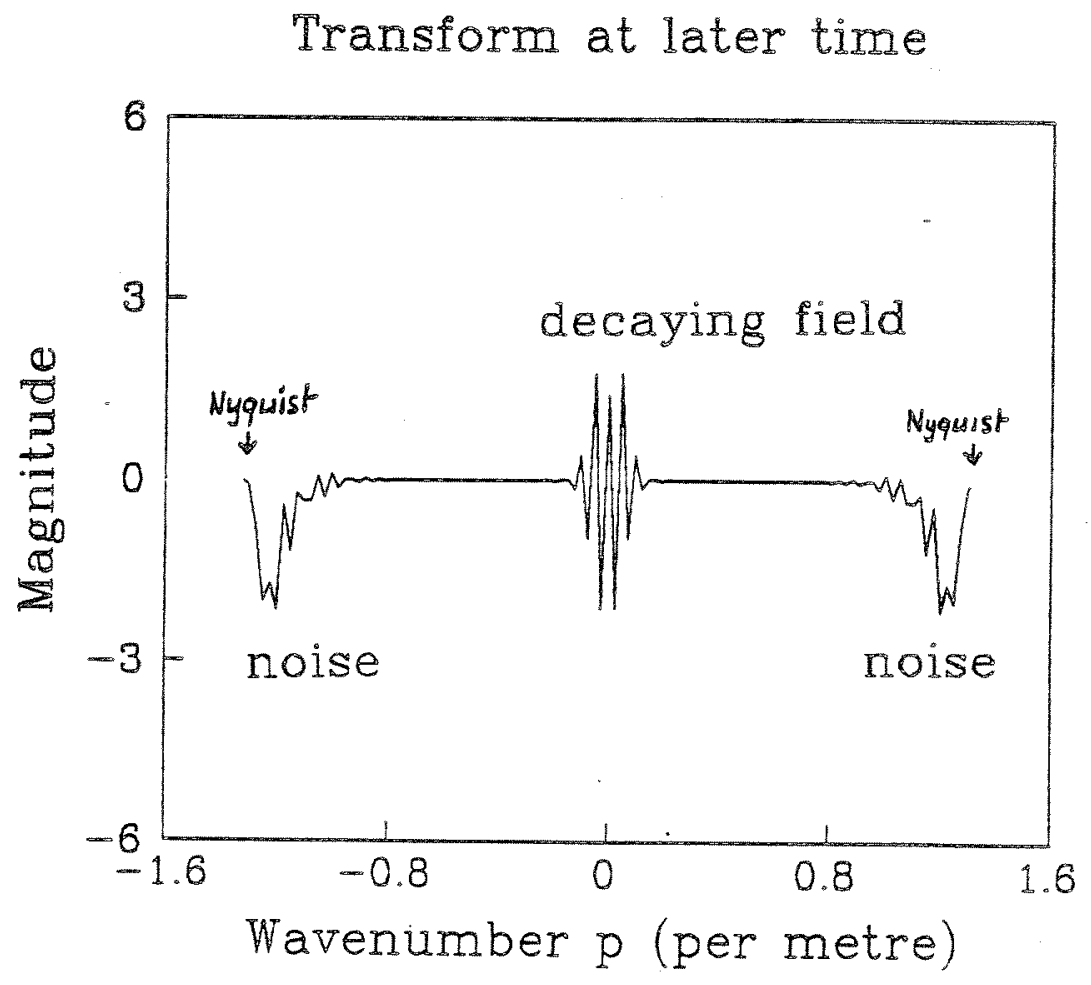

EICURE 4.3 The $p$ axis values for the discrete Fourier transform of the final field (the osclilating field) in
figure 4.1. 
more wavenumbers become negligible. Filtering in a manner such as this suppresses the instabilities seen on figure 4.1. The disadvantage is that the filtering operation takes some extra computer time.

When the grid is expanded the strength of the fields outside the previous discretization area are not known. One possible procedure is to assume that the fields are zero outside the previous grid. The effect of this can be seen on figure 4.4a. The graph shows the decay through time of the vertical magnetic field at the outer edge of the grid. The exciting field is from a 40 metre by 40 metre loop which is at a height of 10 metres above the sheet. The initial grid has nodes every 2 metres, from $-128 \mathrm{~m}$ to $126 \mathrm{~m}$. The earliest delay time is the lower most curve on the figure. At later delay times the field becomes weaker. The early curves - marked ' $A$ ' on the figure are calculated prior to the grid expansion, and thus the field at distances greater than $126 \mathrm{~m}$ from the centre of the loop are outside the discretization grid and are not calculated. Note that when the area added is assumed to have zero field strength high wavenumber oscillations occur around the discontinuity after the grid expansion. Instead of setting the fields to zero outside the initial grid an alternative is to calculate the magnetic field at the delay time of the grid expansion by using the retreating image solution for the uniform thin sheet. As expected the fields in this case are much better behaved (see figure 4.40 ) . 
Effect of increasing the Grid Size
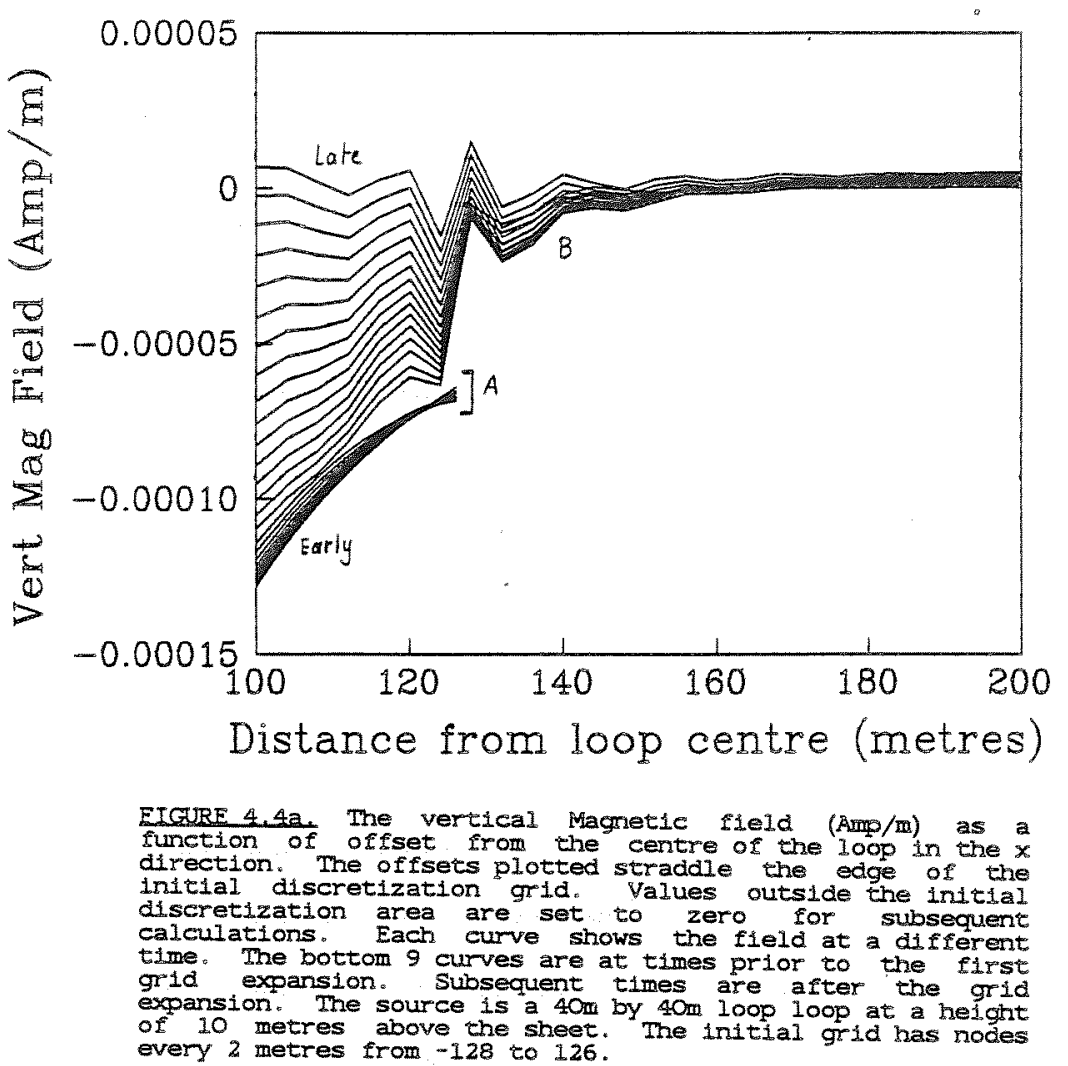

\section{Using the Image solution}

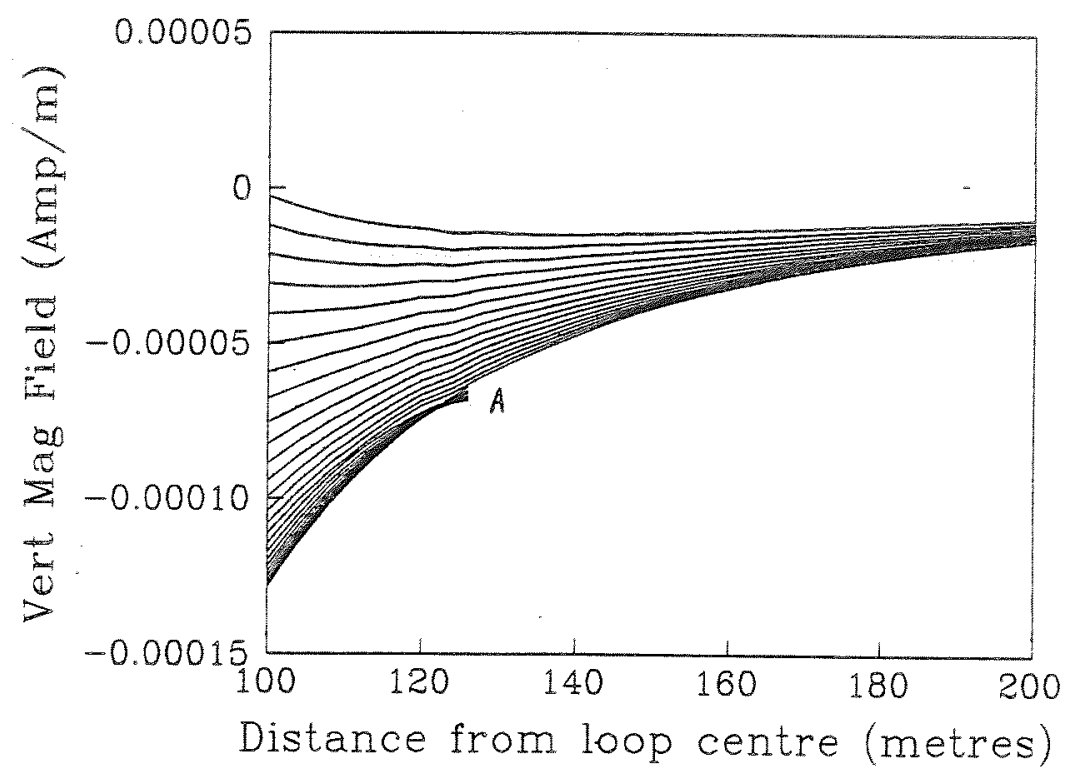

EIGURE 4.40. The vertical Magnetic field (Amp/m) as a function of offset from the centre of the loop in the $x$ direction. The offsets plotted straddie the edge of the initial ciscretization grid. Values outside the intial discretization area are set to the image solution for subsequent calculations. Each curve shows the field at different time. The botton 9 curves are at times prior the first grid expansion Subseouent times are prior to grid expansion. The source is a 40 by heicht of 10 metres able loop loop at a neight of 10 metres above the sheet. The initial grid has 
The field initially is that associated with a single source, however the fields subtracted are calculated using the discrete Eourier transform, and thus repeat periodically in space. 'This multiple loop effect manifests itself near 'A' by the fields actually becoming more negative. However the problem largely corrects itself after the grid has been expanded (see figure $4.4 b)$.

\subsection{Methods of time stepping}

Because the left hand side of equation 4.0 .1 can be calculated explicitly, the equation is an ordinary differential equation of the form

$$
d U / d t=f(U, t) \quad: \quad U=U(t)
$$

A finite difference approximation can be used to approximate this equation. Let the fields at time ' $\mathrm{nh}$ ' (where $\mathrm{h}$ is the step size) be approximated by $U^{n}$. Finite difference methods assume that $U^{n}, U^{n-1}, U^{n-2}, \ldots$ are known, and that the field $U^{n+1}$ at time $(n+1) h$ is required. There are two categories of finite difference methods - implicit and explicit schemes. The implicit schemes use values of ' $f$ ' which depend on $U^{n+l}$. $A$ matrix equation must be solved to calculate the time stepped field $U^{n+1}$, as $U^{n+1}$ appears on the left and right hand side of 4.2.1. If the values of $f$ in 4.2 .1 are independent of $U^{n+1}$ or subsequent values of $U$, then the scheme is an explicit finite difference method. 
As explicit schemes do not require matrix inversions they are normally computationally quicker than the implicit schemes. Thus only explicit methods of finding the fields were examined. The implicit schemes do however have the advantage of being more stable (Lapidus and Pinder (1982), and Richtmeyer and Morton (1967)).

The forward difference (or Euler) scheme can be written

$$
U^{n+1}=U^{n}+h \cdot f^{n}
$$

where

$$
f^{n}=f\left(U^{n} \cdot n h\right)
$$

The truncation error for this scheme is $O(h)$. The order of the truncation error can be improved by using a centred (or two step) finite difference approximation (Mesinger and Arakawa (1976)). The simplest two step scheme is the leap-frog (or central difference) scheme

$$
U^{n+1}=U^{n-1}+2 h \cdot E^{n}
$$

The truncation error is $O\left(h^{2}\right)$.

The dotted Iine on figure 4.5 depicts the vertical magnetic field calculated by stepping the field through time using the leap frog scheme. See the figure caption for details of the conductivity and geometry. The leap-frog solution oscillates about the retreating image solution (solid line). The oscillation is because the solution to the differential equation can be split into two parts--each part consists of a set made up 
The Field at the centre of the loop

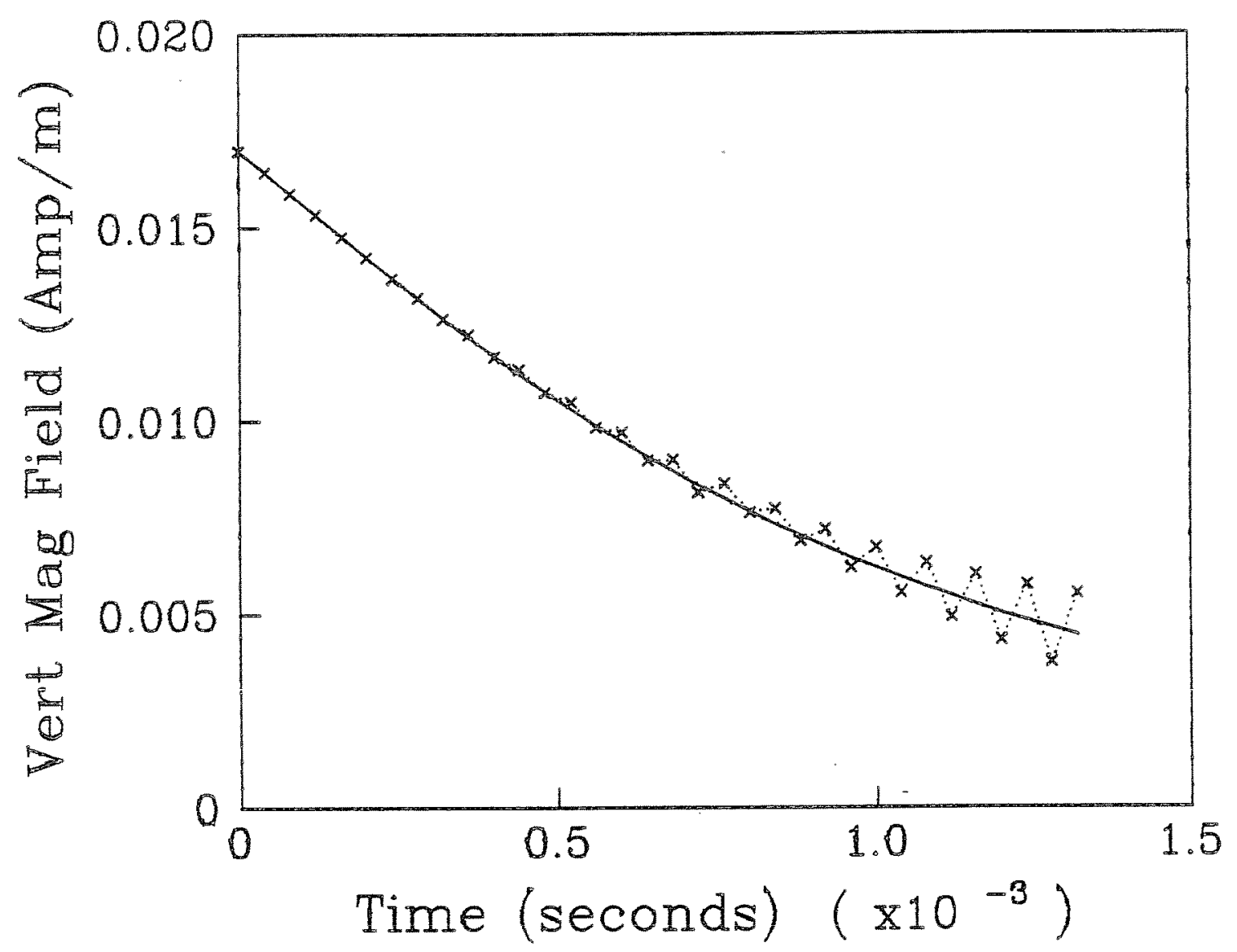

EICURE 4.5 The field as a function of delay time on the sheet $(\delta t=100 S)$ measured directly below the centre of a $40 \mathrm{~m}$ by $40 \mathrm{~m}$ exciting loop source 10 metres above the sheet. Solid line - the image solution. Dotted line - the leap-frog stepped solution. 
of alternate points. These two parts can and do diverge from each other. The divergence is because the derivative calculated from one part is used to calculate the amount to add on to the other part. A small difference between the two parts will result in the two sets of solutions diverging further. It would seem that some type of averaging between these two solutions would overcome this problem. Young (1968) has addressed this problem and the most efficient and the most accurate of the two step schemes he discusses is the Adams-Bashforth scheme

$$
U^{n+1}=U^{n}+h\left(3 f^{n}-e^{n+1}\right) / 2
$$

which is also accurate to $O\left(\mathrm{~h}^{2}\right)$ (Young (1968) and Mesinger and Arakawa 1976). Young also describes a leap-frog scheme which uses a periodic correction:

$$
\begin{aligned}
& \hat{U}^{n-1 / 2}=\left(U^{n}+\dot{U}^{n-1}\right) / 2 \\
& \vec{U}^{n}=\hat{U}^{n-1 / 2}+\hat{f}^{n-1 / 2} h / 2 \\
& \bar{U}^{n-1}=\hat{U}^{n-1 / 2}-\hat{E}^{n-1 / 2} h / 2
\end{aligned}
$$

Where $\vec{U}^{n}$ and $\vec{U}^{n-1}$ are the corrected values. This is also accurate to order $\left(h^{2}\right)$.

Eigure 4.6 shows the percent error obtained when the Adams-Bashforth and "corrected" leap-frog schemes are compared to the image solution. The check point used for comparision is a point on the sheet directly below the centre of the loop. The model is a $40 \mathrm{~m}$ by $40 \mathrm{~m}$ loop initially 1 metre above a sheet with $\mathrm{R}=. \mathrm{OI}$ Ohms. Although the axis has been labelled "time" it can actually be replaced with a variable independent of the value of 


\section{Time stepping methods - check point at $(0,0)$}

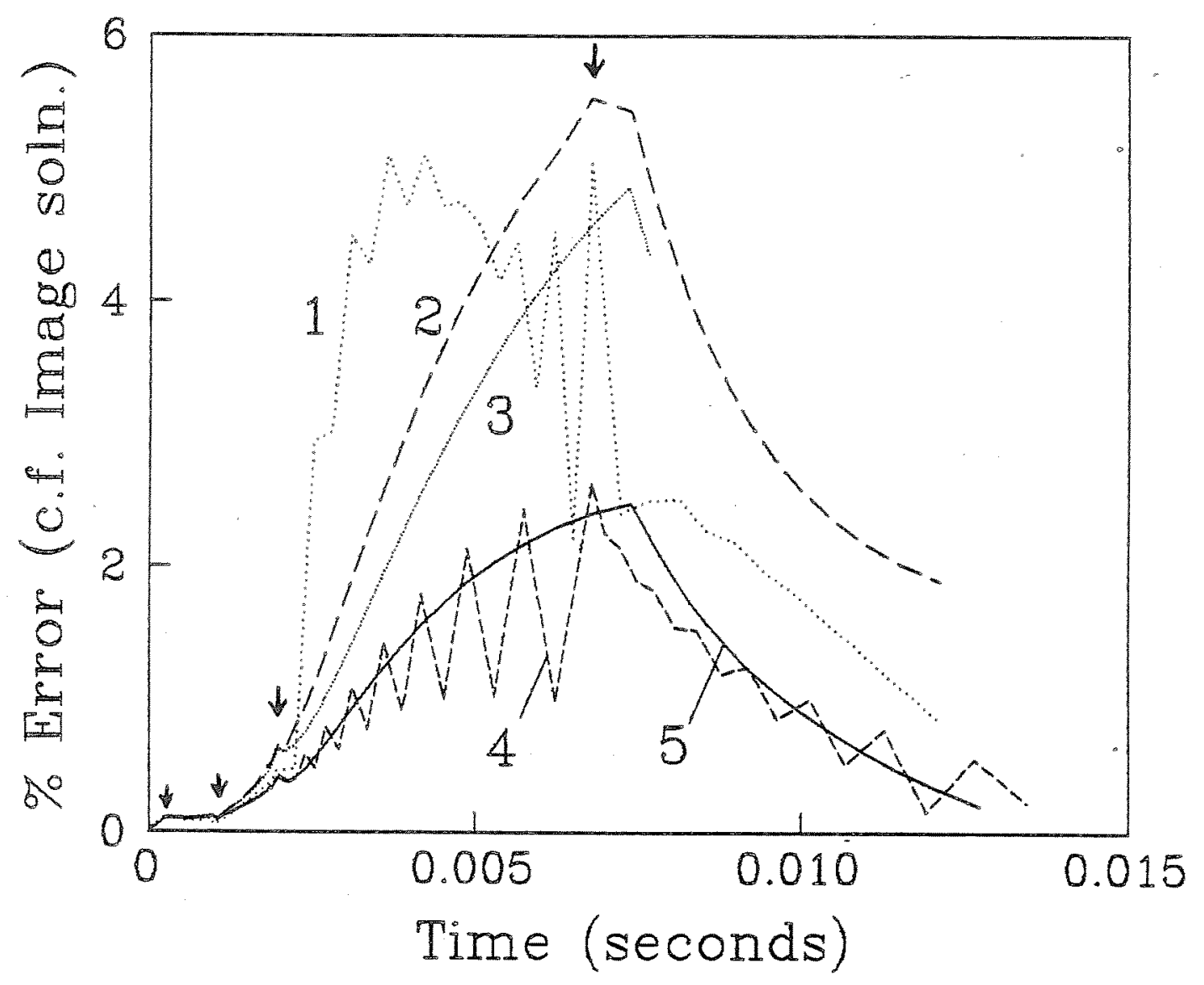

EIGURE 4.6 A comparison of two-step time stepping methods. The solutions are compared against the image solution for a point directly below the centre of the loop $(0,0)$. The loop is $40 \mathrm{~m}$ by $40 \mathrm{~m}$ and $1 \mathrm{~m}$ high. Curves 1,2 and 3 use the Adams-Bash forth scheme with $s=1.0,1.1$, and 1.1, while the time steps between grid expansions is 15,15 and 17 . Curves 4 and 5 are for the corrected leap-frog method and they use $s=1.1$ and the steps per grid expansion are 15 in both cases. The solution is corrected every 15 th step for curve 4 and every step for curve 5 . 
$R$ chosen for these plots. This can be done by multiplying each value of "time" by $2 R / \mu$. The graph is thus applicable for all conductivity thickness products (ot). Curve I is calculated with the Adams-Bashforth scheme, it does not use increasing time steps (i.e. $s=1)$, and the number of time steps between each grid expansion is approximately 15. Curves 2 and 3 have $s=1.1$. In curve 2 the number of time steps between each grid expansion is approximately 15, but in curve 3 it is 17. Using an expanding time step results in the solutions being better behaved. Also using smaller time steps gives a more accurate solution, but the fields have not been calculated out to such a late delay time in the case of curve 3 as they have in other cases (the number of time steps were restricted to 100). Curves 4 and 5 are calculated with the corrected leap-frog scheme, and the errors are less. The fourth curve has been corrected every 15th step, while the fifth curve has been corrected at each step. The computing time for the corrected leap-frog method increases as the number of time steps between corrections decreases. For example for the fifth curve the computation time is 1 hour 50 minutes compared with curve 3 which took 47 minutes (on a VAX 11/780). The approximate times of the grid expansions (they vary from one time stepping method to the next) is shown with arrows on figure 4.6 .

The choice of time stepping scheme will depend on the priorities of the user. If a very accurate answer is required, then one of the slower schemes, with many time steps would be appropriate however for a quick idea of the fields one of the less accurate schemes would be more appropriate. At the delay times for which the errors are greatest (near .007 seconds) the 
field is less that one hundredth of the initial field at $t=0$, and in many circumstances this error would be tolerable. Note also that after. 007 seconds the errors again decrease. This is because the grid has been expanded at this delay time.

When the ratio $1 / \mathrm{b}$ (equation 4.1 .4 ) oscillates in magnitude, instabilities similar to those on figure 4.1 result. It was observed that in many cases the oscillations did not develop when the step size was decreased. The fact that the growth of the errors can be monitored in such a manner is encouraging and this could provide a means of ensuring that the errors do not grow without bound for the variable conductivity case--for which no numerical checks are available. Note that an inappropriate time step size is not the only source of error. Errors can also be attributed to inadequate sampling in the space domain.

Percent errors were also plotted for check points not at the centre of the loop, and these are seen on figure 4.7. Once again a $40 \mathrm{~m}$ by $40 \mathrm{~m}$ loop was placed I metre above a sheet with is centre at the origin of a grid $127 \mathrm{~m}$ by $127 \mathrm{~m}$. The check points used are $(0,0)$ - graph I, $(0,-32)$ - graph 2, (-32,-32) - graph 3. $(0,-64)$ - graph 4 and $(-64,-64)$ - graph 5. The errors are large when the check points are near the edge of the grid graphs 4 and 5 . Here the fields are about 20 percent in error prior to the first grid expansion. This effect is due to the multiple sources discussed at the end of section 4.1. The effect of these errors can be minimized by a wise choice of grid size and loop placement, and by keeping the areas where accurate answers are required away from the edge of the grid. The other spikes on the graphs occur where the magnetic field changes 


\section{Different Check points}

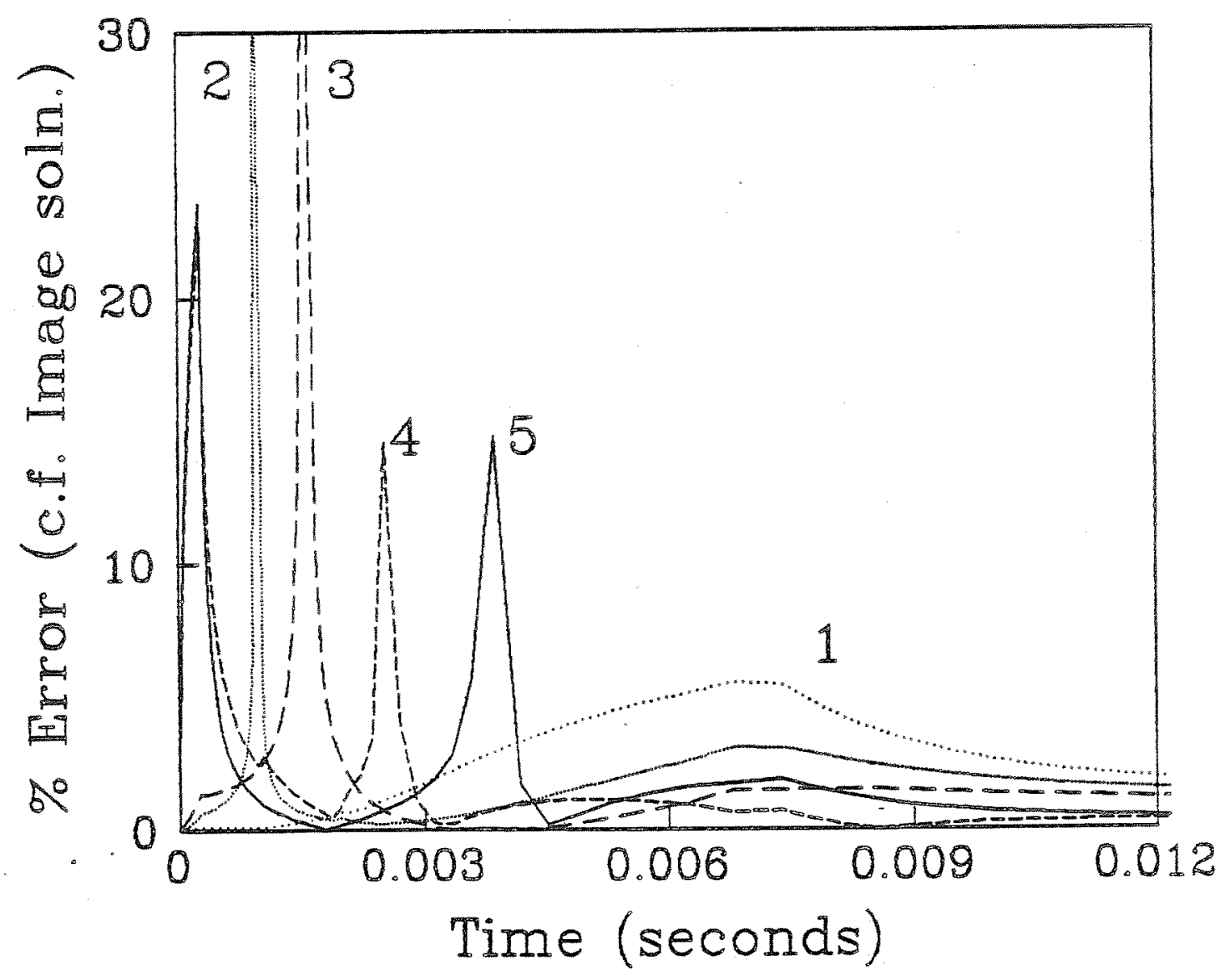

EIGURE 4.7 The percent error obtained as a function of time using the Adams-Bashforth scheme for different check points. $s=1.1$ and 15 steps between grid expansions were the parameters used. The exciting source was $40 \mathrm{~m}$ by $40 \mathrm{~m}$ Im high and centred at $(0,0)$. The initial grid was $127 \mathrm{~m}$ by $127 \mathrm{~m}$. The check points used are $(0,0)$ - graph $1,(0,-32)$ - graph 2. $(-32,-32)$ - graph $3,(0,-64)$ - graph 4 and $(-64,-64)$ graph 5 .

The grid expansions are at approximately the same times as in figure 4.6 
sign. At such points both the image solution and the time stepped solution are small, so although the normalized error is large the magnitude of the discrepency is small. The problem is therefore not critical.

Young (1968) also looked at many one step schemes for which more than one time derivative is evaluated at each iteration. He concluded that two schemes stood out as being more efficient and accurate. His method ' $D$ ', which he concluded was one of the best, cannot be applied to the thin sheet induction problem. The scheme would require that the values of magnetic field on the grid already obtained in that iteration be used to obtain the value at the next grid point in the same iteration. This would require transformation to and from the wavenumber domain so as to get an updated time derivative. This is exceedingly inefficient. The other scheme (Young's method A), and two of the one step schemes discussed in Mesinger and Arakawa (1976) were applied to this problem. One is the backwards-Euler scheme:

$$
\begin{aligned}
& U^{n+1}=U^{n}+h \cdot f^{n} \\
& U^{n+1}=U^{n}+h \cdot e^{n+1}
\end{aligned}
$$

where $U^{n+1}$ is a provisional value used to obtain

$$
f_{n+1}^{*}=f\left(U^{n+1},(n+1) h\right) .
$$

This scheme is accurate to order (h). Then there is the Heun (double forward) scheme: 


$$
\begin{aligned}
& U^{n+1}=U^{n}+h \cdot f^{n} \\
& U^{n+1}=U^{n}+(h / 2)\left[f^{n}+e^{n+1}\right]
\end{aligned}
$$

which is accurate to order $\left(h^{2}\right)$, and Young's Method A:

$$
\begin{aligned}
& U^{U_{n+1 / 2}}=U^{n}+h / 2 f^{n} \\
& \hat{U}^{n+1 / 2}=U^{n}+h / 4\left[f^{n}+f^{n+1 / 2}\right] \\
& U^{n+1}=U^{n}+h \cdot \hat{E}^{n+1 / 2}
\end{aligned}
$$

which is accurate to order $\left(\mathrm{h}^{2}\right)$. These methods have been applied to the problem, and the error between the time stepped solution and the image solution have been plotted in figure 4.8 . At each step the time increment was expanded by a factor of 1.1. and 15 time steps were used between each grid expansion. The backward-Euler scheme (Iabel 1) gives very poor results. The Heun scheme (label 2) gives results which are similar to the leap-frog scheme corrected at each step. This is not surprising, as the two schemes are similar - both involving two steps of length $\mathrm{h} / 2$. Young's method A (label 3) however stands out as being better than any other scheme. From equations 4.2.8 it can be seen that Young's method involves three calculations of the time derivative, and thus takes more computer time. A comparision of the accuracy of two schemes when compared on an equal computation time basis is shown on figure 4.9. As Young's method A takes approximately twice as long as the Adams-Bashforth scheme the step size used for Young's method A 
One Step methods - check point at $(0,0)$

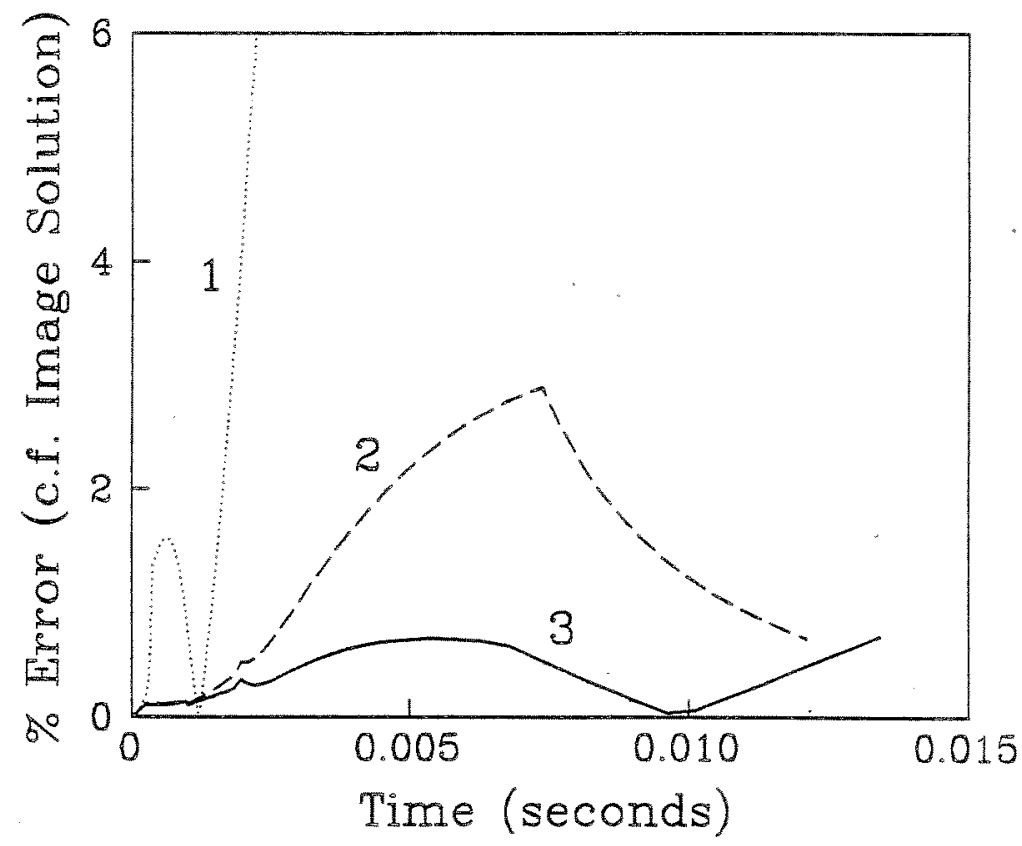

EIGURE 4.8 A comparison of one-step time stepping methods. The solutions are compared against the lmage solution for a point directly below the centre of the loop $(0,0)$. The 100 p is $40 \mathrm{~m}$ by $40 \mathrm{~m}$ and $1 \mathrm{~m}$ high. Curve 1 is for the backwards-Euler scheme, curve 2 is for the Heun scheme and curve 3 is for Youngs method ' $A$ '. In each case $s=1.1$ and the time steps between grid expansions is 15

Adams-Bashforth and Young schemes

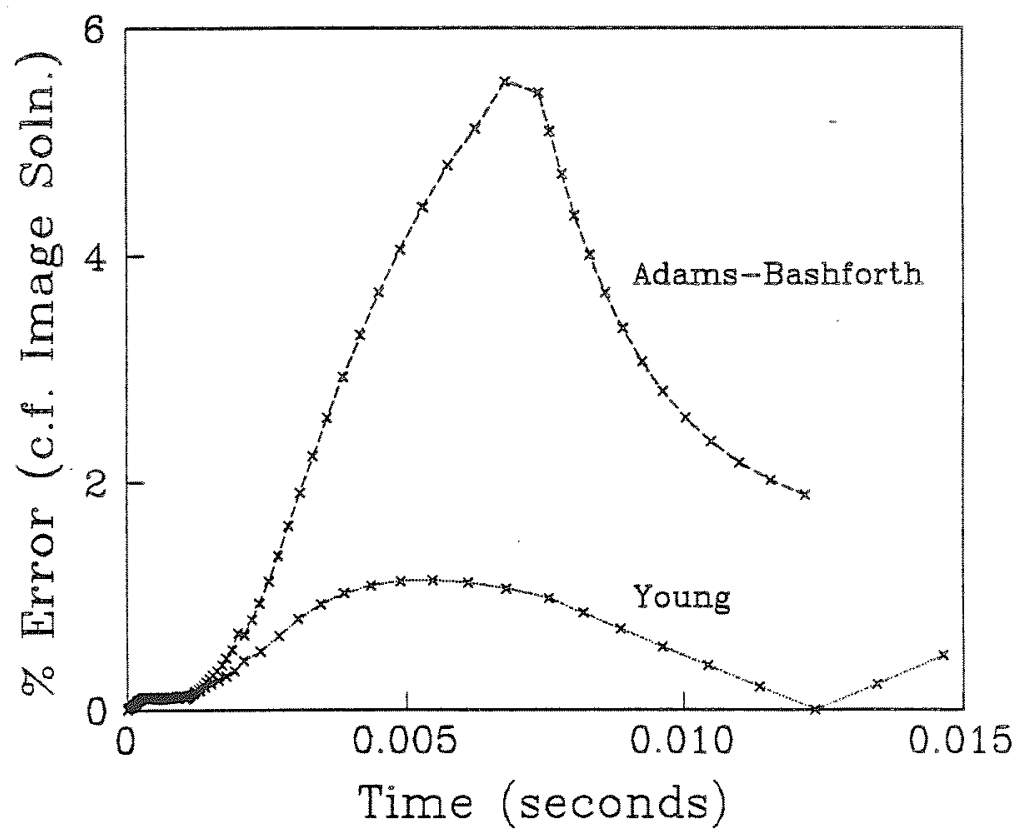

EIGURE 4.9 A comparison of the percent error when equal comoutation times were used to step the same total time. Young's method $A$ and the Adams-Bashforth scheme were Young's method $A$ and the Adams-Bashforth scheme were
compared. The model is the same as figure 4.6. The ' $X$ 's on the plots show the times at which the fields were calculated. 
was seven time steps per grid expansion, and for the Adams-Bashforth scheme fifteen time steps per grid expansion. Clearly Young's method A is superior.

For Young's method ' $A$ ' the optimal number of time steps to use per grid expansion was obtained by observing the convergence of the solutions when plotted as a function of decreasing step size (see figure 4.10). Each curve represents the error at a different delay time. From the graph there appears to be no need to use more than about 10 time steps per expansion, and 6 steps may be tolerable if the fields are only required at early delay times. Similar results for the Adams-Bashforth scheme (figure 4.11) suggest that 16 time steps per grid expansion is optimal, but as few as 10 may be used if accurate answers at early time only are required. It was found that answers were accurate until instabilities developed.

\subsection{The Variable Conductivity Case}

When the conductivity varies within the sheet the methods of calculating the response are similar to those discussed in section 4.1, except that the terms involving the derivatives of $R$ and the horizontal components of the magnetic field must be included. Because the horizontal components of the field must be calculated in Eourier transform space, then transformed back to space domain, the computation time for each iteration increases by a factor of about two.

As for the uniform thin sheet case the grid is expanded when the amount of information in the high wavenumbers becomes negligible. When the grid is expanded, the fields outside the 
Young's method A - at different times

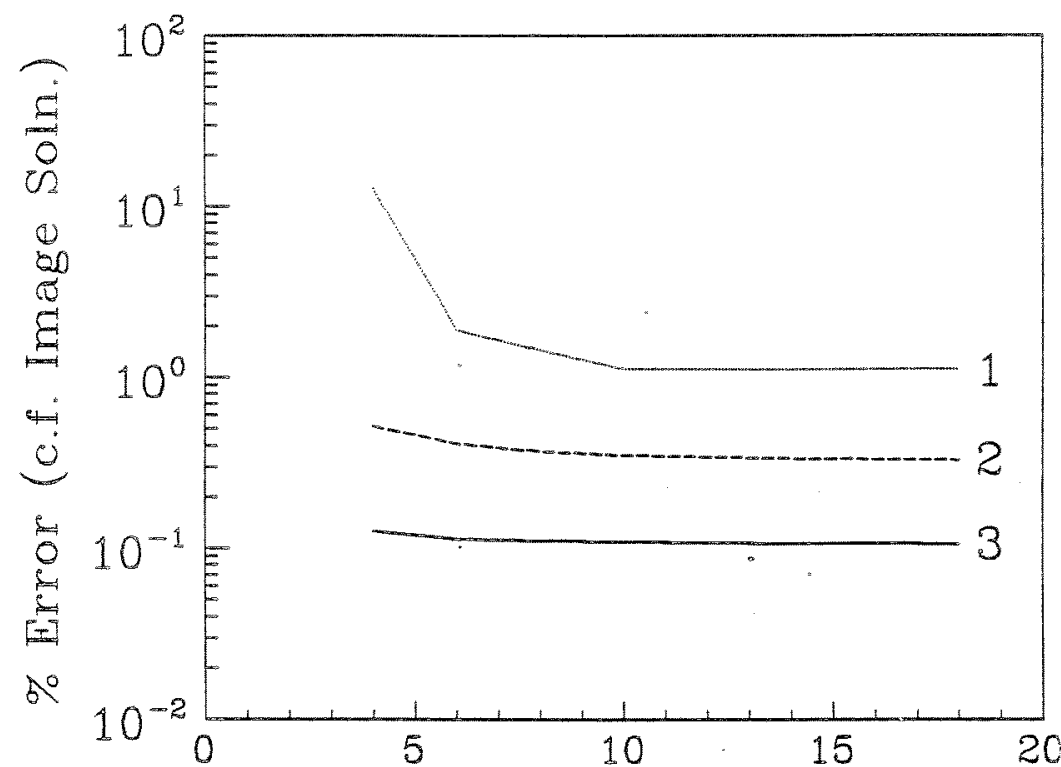

No. of time steps per grid expansion

EIGURE 4.10 The percent error between Young's method A and the image solution compared against the image solution at different times (curve $1-5.5$ msec, curve 2 . 20 msec, curve 3 -. 3msec) plotted as a function of decreasing step size. $\sigma t=100 \mathrm{~S}$, and a point on the sheet below the centre of a 40 by $40 m$ in 1000 in above the sheet was again used.

The Error at different times

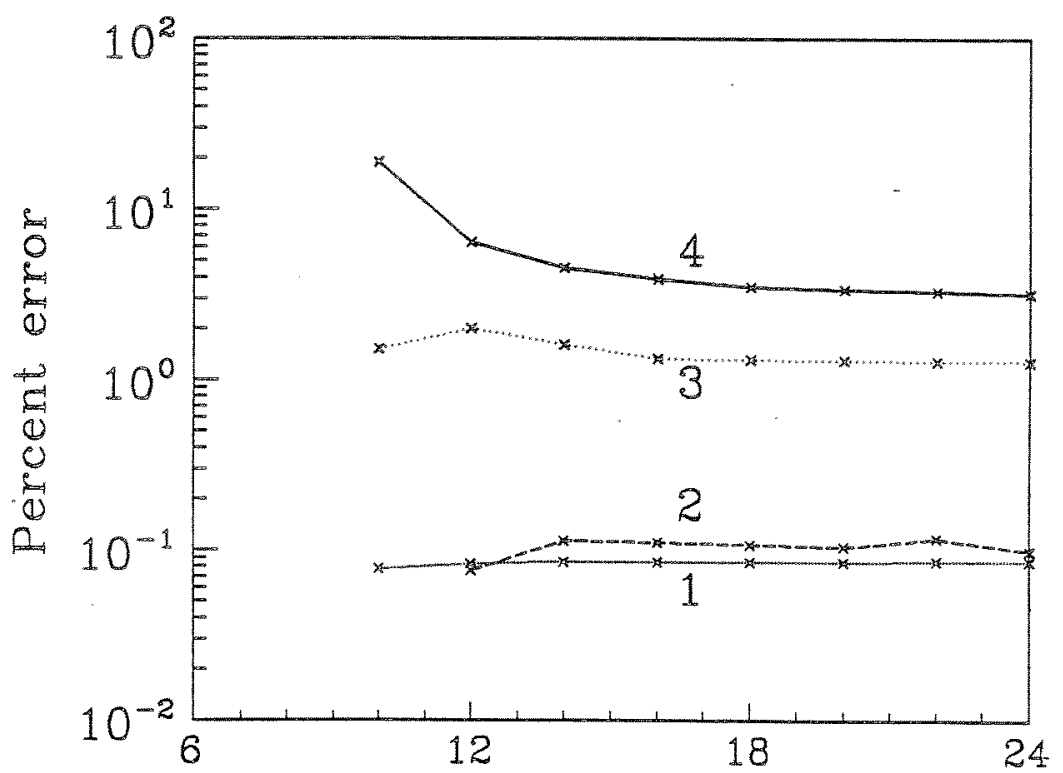

No. of steps between grid expansions

EIGURE 4.11 The percent error between the Adams-Bashforth scheme and the image solution compared against the image solution at different times (curve $1-.2 m s e c$ curve $2-1$ msec, curve 3 - 3 msec, curve $4-8$ msec) plotted as a function of decreasing step size. $\delta t=100 \mathrm{~s}$, and a point on the sheet below the centre of a $40 m$ by $40 \mathrm{~m}$ loop Im above the sheet was again used. 
area of discretization must be estimated, otherwise the problems illustrated in figure 4.4 will arise. In the previous section, the image solution was used. In calculating the image solution a resistance for the sheet outside the area of discretization must be assumed. A simple approach is to assign a background resistance value. The anomalous resistivities within the initial discretization grid will differ from this value. When the grid is expanded, this background resistance value can be used to calculate the image solution at points outside the grid for the delay time ' $t$ ' at which the grid is expanded.

If more complicated resistance distributions outside the initial area of discretization are required, then more elaborate solutions should be used. These would also involve some approximation.

The assumption that the resistance outside the sheet is a constant is not too restrictive, as the initial area of discretization should contain the areas of interest, and the resistance values near the edge of the sheet should be equal to the background resistance.

In section 4.1 equation 4.1 .5 was used to calculate the time step size. The equation is no longer valid for the variable conductivity case, but it can still be used to provide an estimate of the size of the time step.

For the constant conductivity case, the magnitude of the wavenumber components in the transform of the field decay away exponentially at rates that depend upon the resistance. It is therefore reasonable to assume that if a sheet is made up of a number of areas of different resistance, then the components 
relating to those areas will decay approximately exponentially, but at different rates. Thus, as the delay time increases, a bimodal or multimodal distribution of the wavenumber components of the fields may develop and the grid must not be expanded until the components associated with the higher wavenumber variation have decayed away. The grid is still expanded once the wavenumber components at half the Nyquist become negligible although this may not occur as soon as it would in the uniform sheet case. Equation 4.1 .5 predicts the delay time at which the grid expansion should occur for the uniform conductivity sheet case. If instabilities in the variable conductivity sheet case developed after this delay time then a grid expansion was forced to take place. In the cases tested, this restored stability. When an expansion is forced the solution is being pushed towards the constant conductivity case. This is because i) the image solutions are covering a larger percentage of the gridded area, and $i i)$ the predicted delay time of the grid expansion is the delay time that a grid expansion would take place if the sheet was a uniform sheet. Instabilities can also be suppressed by filtering. Filtering and forcing an expansion throws away information in the wavenumber components that are not present in the uniform thin sheet solution, and thus the variable thin sheet solution will become more like the uniform sheet solution.

The methods presented in this section are designed to calculate the effect of overburden of gently varying conductivity. Therefore forcing the solution towards the solution for the uniform sheet is not considered to be too restrictive. 


\subsubsection{Model Runs}

\subsubsection{Checks against the Uniform Sheet Case}

Eigure 4.12 shows the results obtained when the variable conductivity program is applied to the uniform sheet problem. Compare these plots with those on figure 4.9. The results agree sufficiently to say that the programs are consistent. Computation times were one hour for the Adams-Bashforth scheme, and two hours for the Young scheme (VAX 11/750). Eewer time steps have been used in these cases than the cases for which computational run times have been quoted previously.

\subsubsection{A Resistive Inhomogeneity}

Figure 4.13 shows a contour map of the resistance structure within the initial grid of discretization ( $127 \mathrm{~m}$ by $127 \mathrm{~m}$ ). The position of the loop is shown on the map. The background resistance is $R=0.01$ Ohms. Within the inhomogeneity $R$ increases gradually up to .037 Ohms. Young's method ' $A$ ' is used with 15 time steps per grid expansion, and the time step expansion factor is 1.1. Profiles of the vertical magnetic field parallel to the $x$ axis, and across the centre of the loop and resistive inhomogeneity are shown on figures 4.14 to 4.17 . The largest amplitude field is that at the earliest delay time, and the amplitude decreases as the decay time increases. On the right hand side of the loop over the resistive inhomogeneity the maximum current (represented roughly by the zero crossing of the vertical component of the magnetic field) moves more rapidly across the resistive inhomogeneity than across the rest of the sheet. Once across the inhomogeneity, it moves out more slowly. 


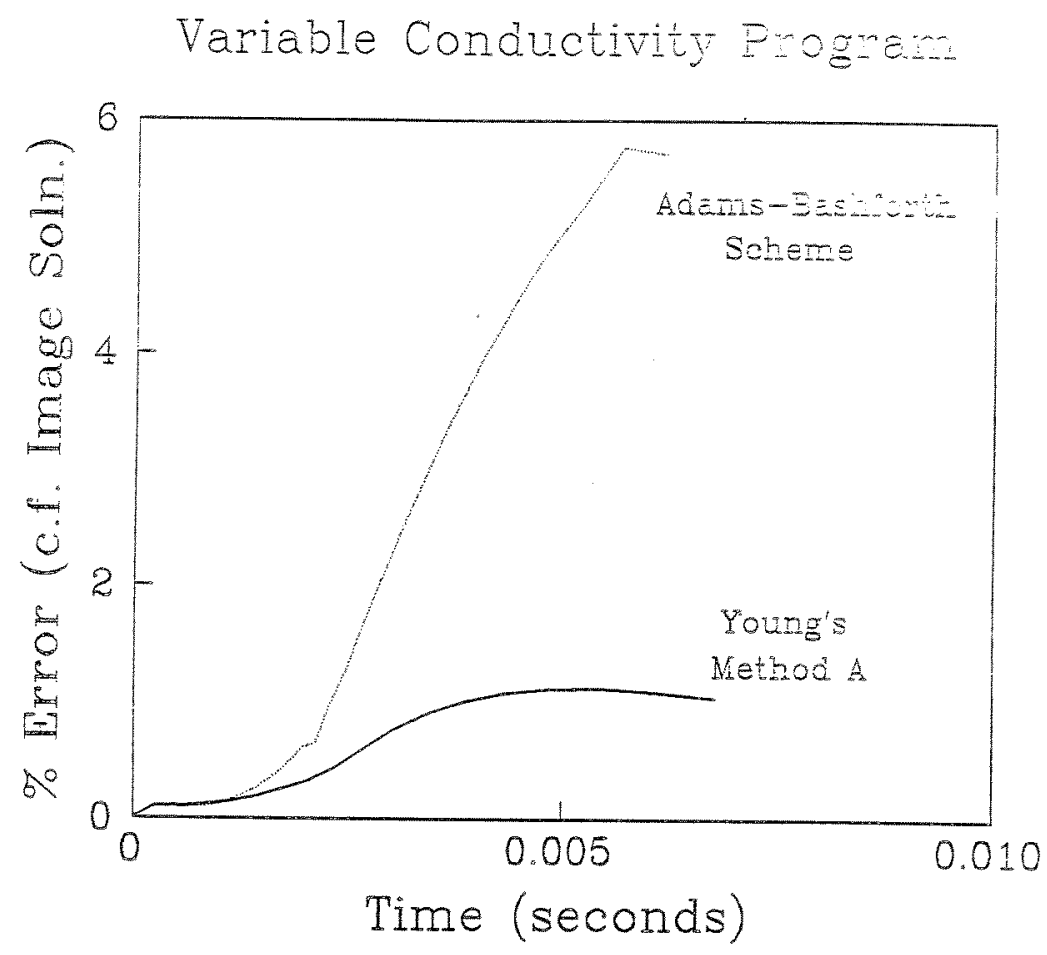

EIGURE 4.12 The percent error between the image solution and the Adams-Bashforth scheme and Young's method A. (C.F. Image solution) for the parameters of figure 4.6 as calculated by the variable conductivity program.

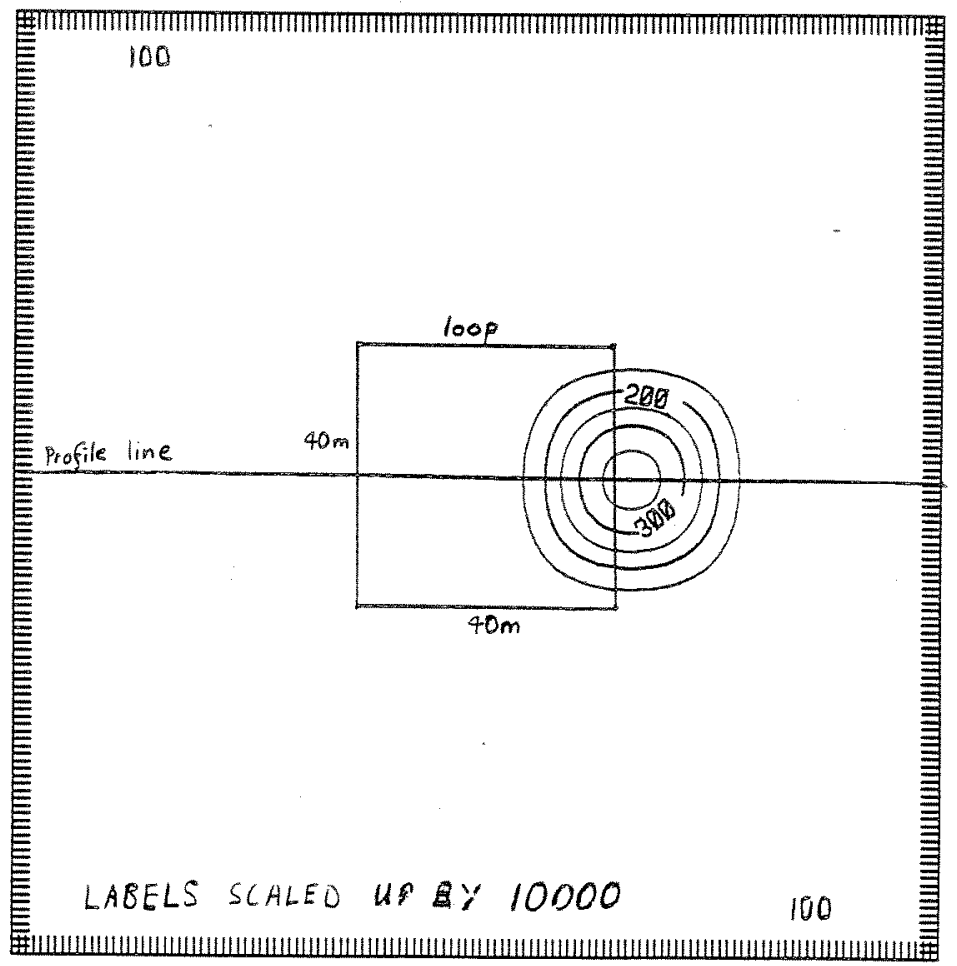

EIGURE 4.13 A contour map of the resistivity structure within the initial grid of discretization (127m by $127 \mathrm{~m}$ ). The position of the loop is shown on the map. The background resistivity is $R=0.01$ Ohms. The contour labels have been scaled up by: 10000 . 
Resistive Inhomogeneity

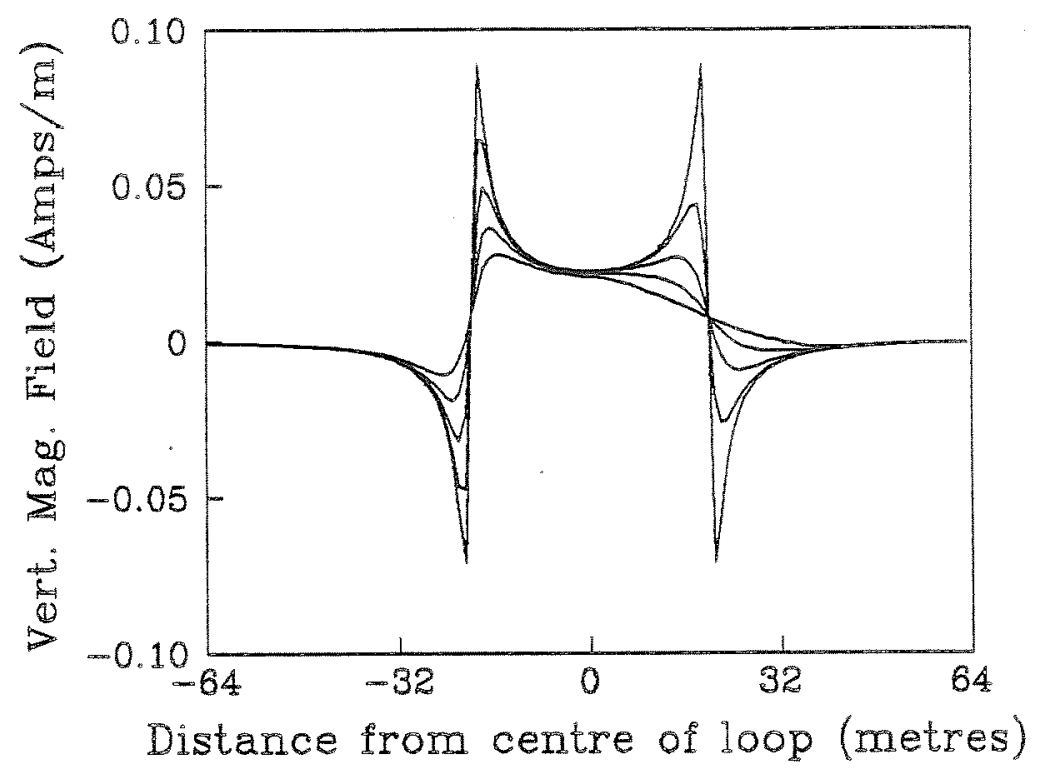

FIGURE 4. 14 The decay of the vertical magnetic field within the initial grid of discretization for the resistive inhomogeneity, grid and source case described in section 4.3.1.2. The larger the amolitude of the plots the earlier the delay time. The delay times on this plot are: $0 . .022$. $.062 . .121$, and 208 , milisseconds.

\section{Resistive Inhomogeneity}

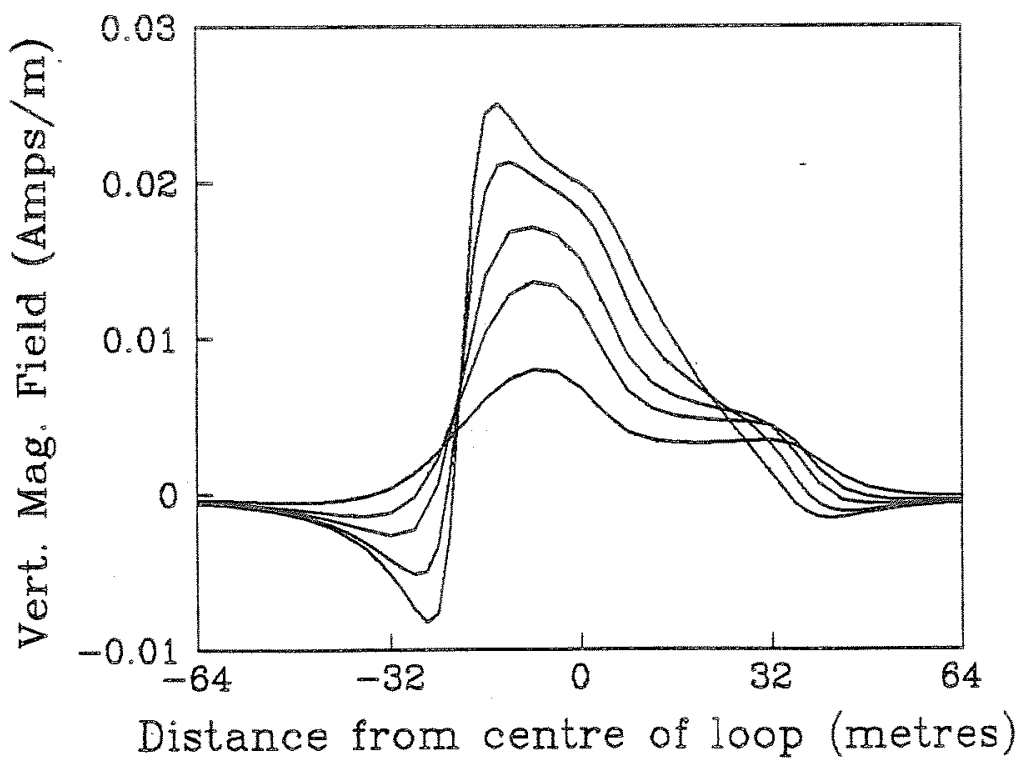

EIGURE 4.25 The decay of the vertical magnetic field within the initial grid of discretization for the resistive inhomogeneity, grid and source case described in section 4.3.1.2. The larger the amplitude of the plots the earlier the delay time. The delay times on this plot are:.257, $.355, .527, .725$, and $1.150 \mathrm{mill}$ iseconds. 
Resistive Inhomogeneity

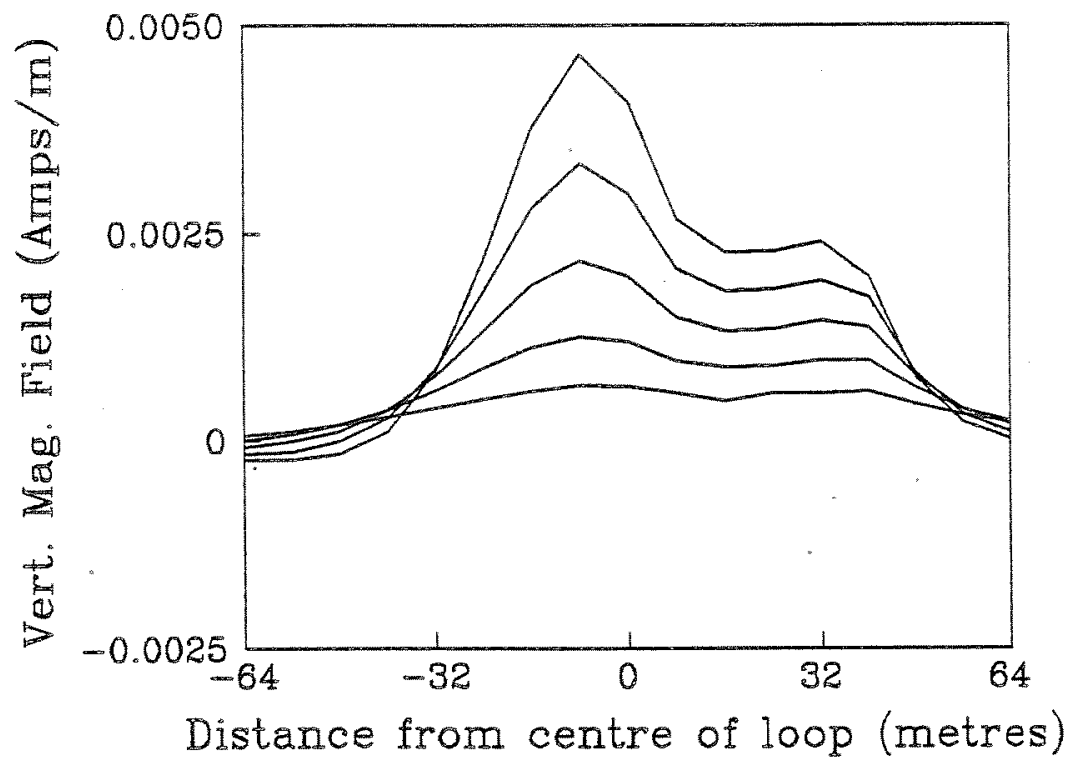

EIGURE 4.15 The decay of the vertical magnetic field within the initial grid of discretization for the resistive inhomogeneity, grid and source case described in section 4.3.1.2. The larger the amplitude of the plots the earlier the delay time. The delay times on this plot are: 1.595. $1.892,2.325,2.961$ and 3.891 milliseconds.

\section{Resistive Inhomogeneity}

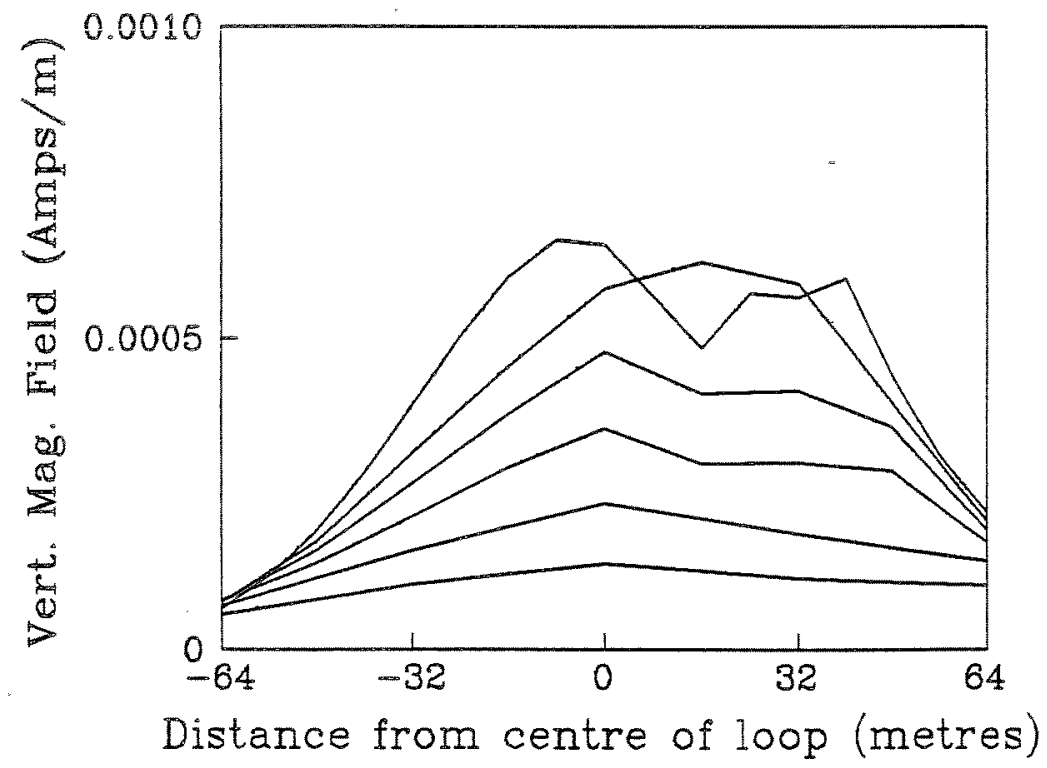

EICURE 4.17 The decay of the vertical magnetic field within the initial grid of discretization for the resistive inhomogeneity, grid and source case described in section 4.3.1.2. The larger the amplitude of the plots the earlier the delay time. The delay times on this plot are: 3.891 . $4.405,4.817,5.420,6.307$, and 7.608 milliseconds. 
The "dual ridge" nature of the profiles in figures 4.15 and 4.16 suggest a partial bifurcation of the current or at least a relative low in the current density. The single steep section on the left hand side is associated with the main current out of the page, however there are two steep parts to the profile on the right hand side of the graph. The left most one occurs to the left of the resistive inhomogeneity, while the one on the right is associated with the zero crossing, and represents the major component of current into the page. These results suggest that the current going into the page is being channelled so as to keep it on the left hand side of the resistive inhomogeneity. The "partial bifurcation" continues until the very latest delay times shown on figure 4.17 where the field is about one thousandth of the initial value. At these delay times there are only 4 points across the profile, where initially there were 128. and it is not expected that the inhomogeneity will have much effect as its size is smaller than the grid sampling interval.

The currents induced around the loop are best observed by looking at contour maps of the stream potential (see figures 4.18 to 4.25). Note that in the final contour map the contours have been multiplied by a scaling factor of 10000 . The currents clearly move more rapidly through the resistive part. In the last plot the effect of the resistive inhomogeneity is not obvious, and this is because i) the fields have been forced towards the uniform sheet solution, and ii) the resistive inhomogeneity is no longer seen in the discretization. 
Page $41 a$

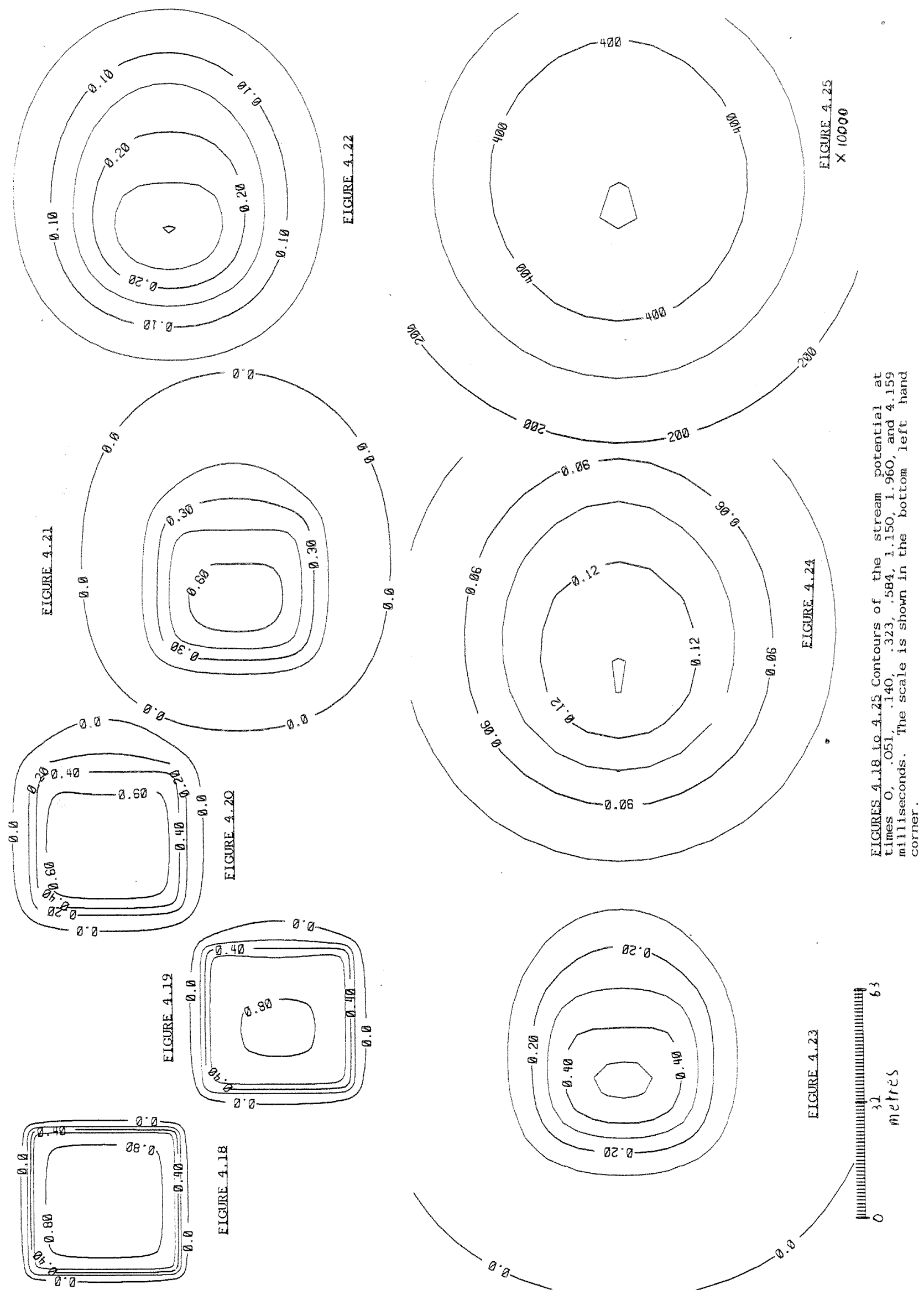




\subsubsection{A Conductive Inhomogeneity}

Eigure 4.26 shows a contour map of a conductive structure within the initial grid of discretization. Again the position of the loop is shown on the $(127 \mathrm{~m}$ by $127 \mathrm{~m})$ map. The background resistance is $\mathrm{R}=0.04$ Ohms. Within the inhomogeneity $R$ decreases gradually down to .015 Ohms. The time stepping method is the same as the previous section. Profiles of the vertical magnetic field parallel to the $x$ axis, and across the centre of the loop and conductive inhomogeneity are shown on figures 4.27 and 4.28. The fields near the conductive area decay less quickly, and the zero crossing moves more slowly through the inhomogeneity. Contour plots of the stream potential - figures 4.29 to 4.36 - illustrate the fact that the currents are better preserved in the conductive area.

\subsection{Accuracy Tests}

There are no other numerical methods which calculate the time decay of the fields for a three dimensional source above a sheet of variable conductivity thickness product. Oristaglio and Hohmann (1984) have calculated the currents induced in a two dimensional earth when excited by line sources, but this cannot be used to check the thin sheet solutions. The other available checks are against scale model experiments, or checks of internal consistency. 


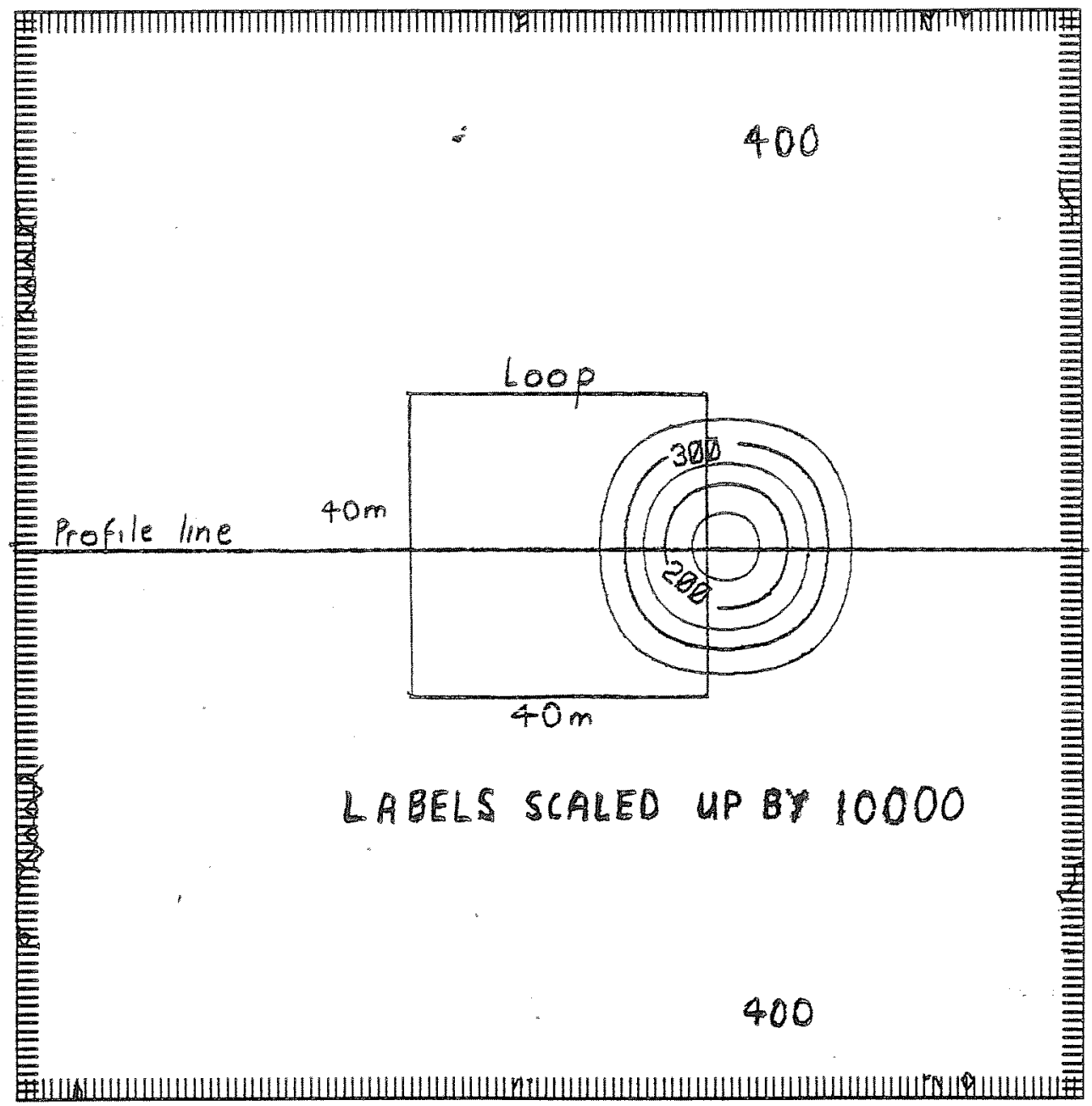

EIGURE 4.26 A contour map of the resistivity structure within the initial grid of discretization (127m by 127m). The position of the loop is shown on the map. The background resistivity is $R=0.04$ Ohms. The contour labels have been scaled up by 10000 . 
Conductive Inhomogeneity

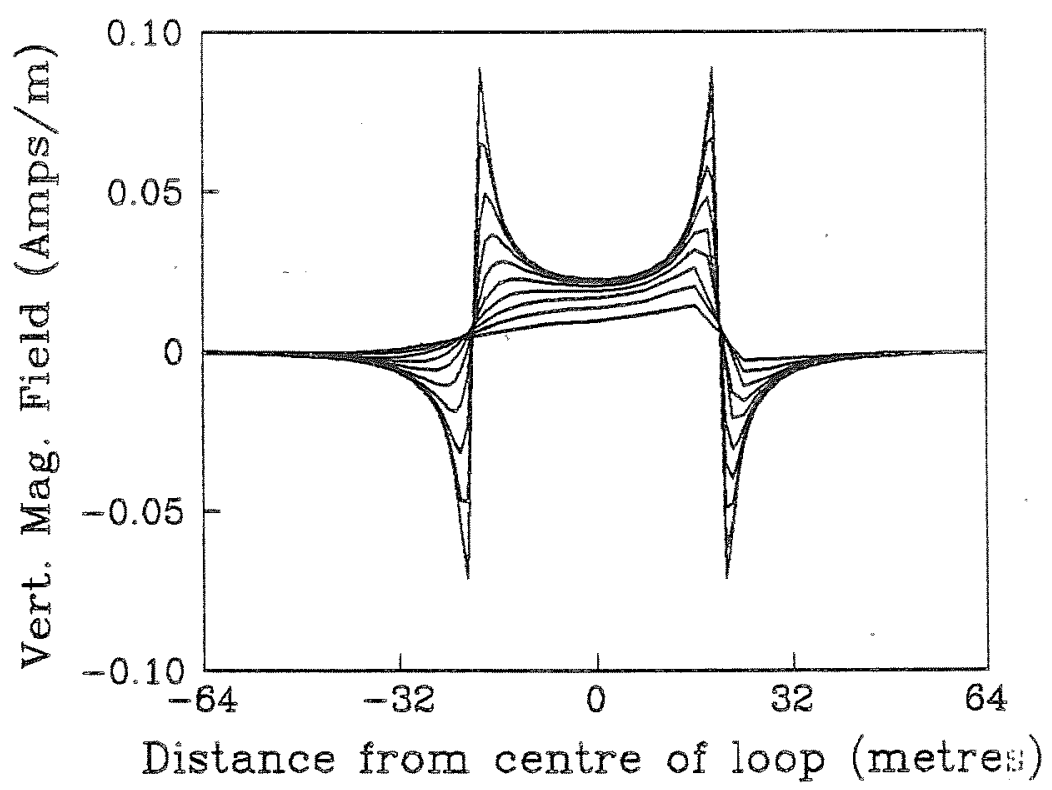

EIGURE 4.27 The decay of the vertical magnetic field within the initial grid of discretization for the conductive inhomogeneity, grid and source case described in section 4.3.1.3. The larger the amolitude of the plots the earlier the delay time. The delay times on this plot are: $0 . .005$. $.016, .030, .052, .080 . .117, .160, .223$ and .315 miliseconds

Conductive Inhomogeneity

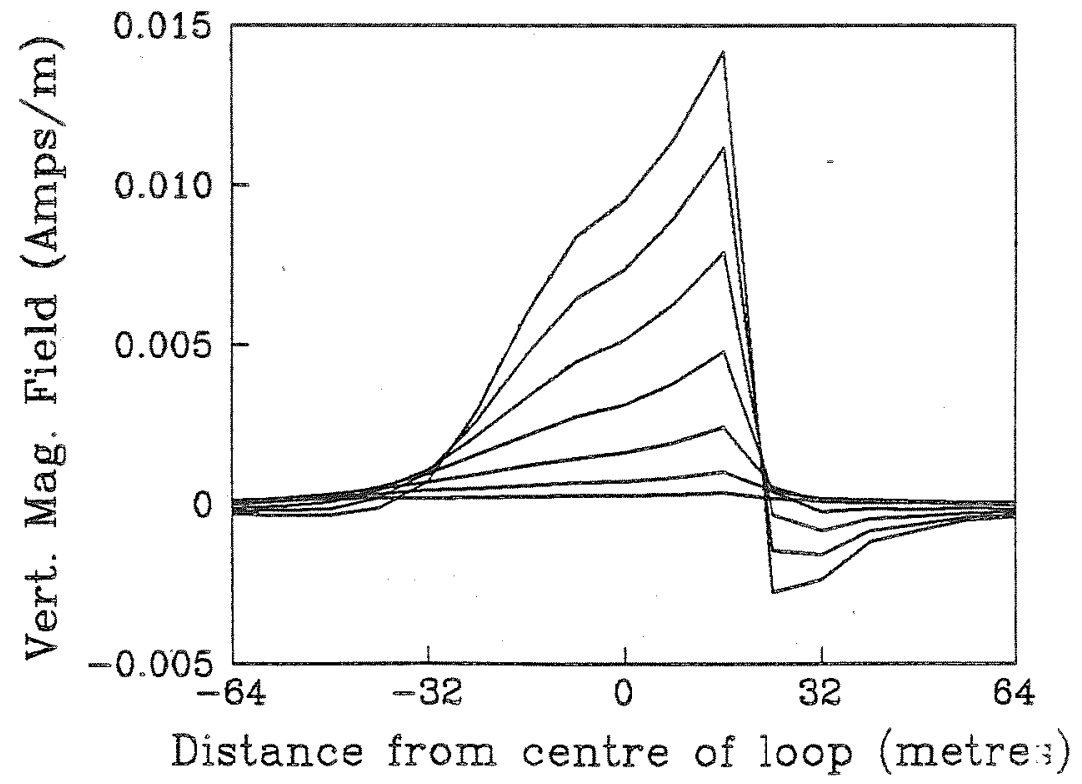

EIGURE 4.28 The decay of the vertical magnetic field within the initial grid of discretization for the conductive inhomogeneity, grid and source case described in section 4.3.1.3. The larger the amplitude of the plots the earlier the delay time. The delay times on this plot are: .315, $.381, .478, .620, .827,1.131$, and $1.576 \mathrm{milliseconds.}$ 
Page $42 \mathrm{c}$

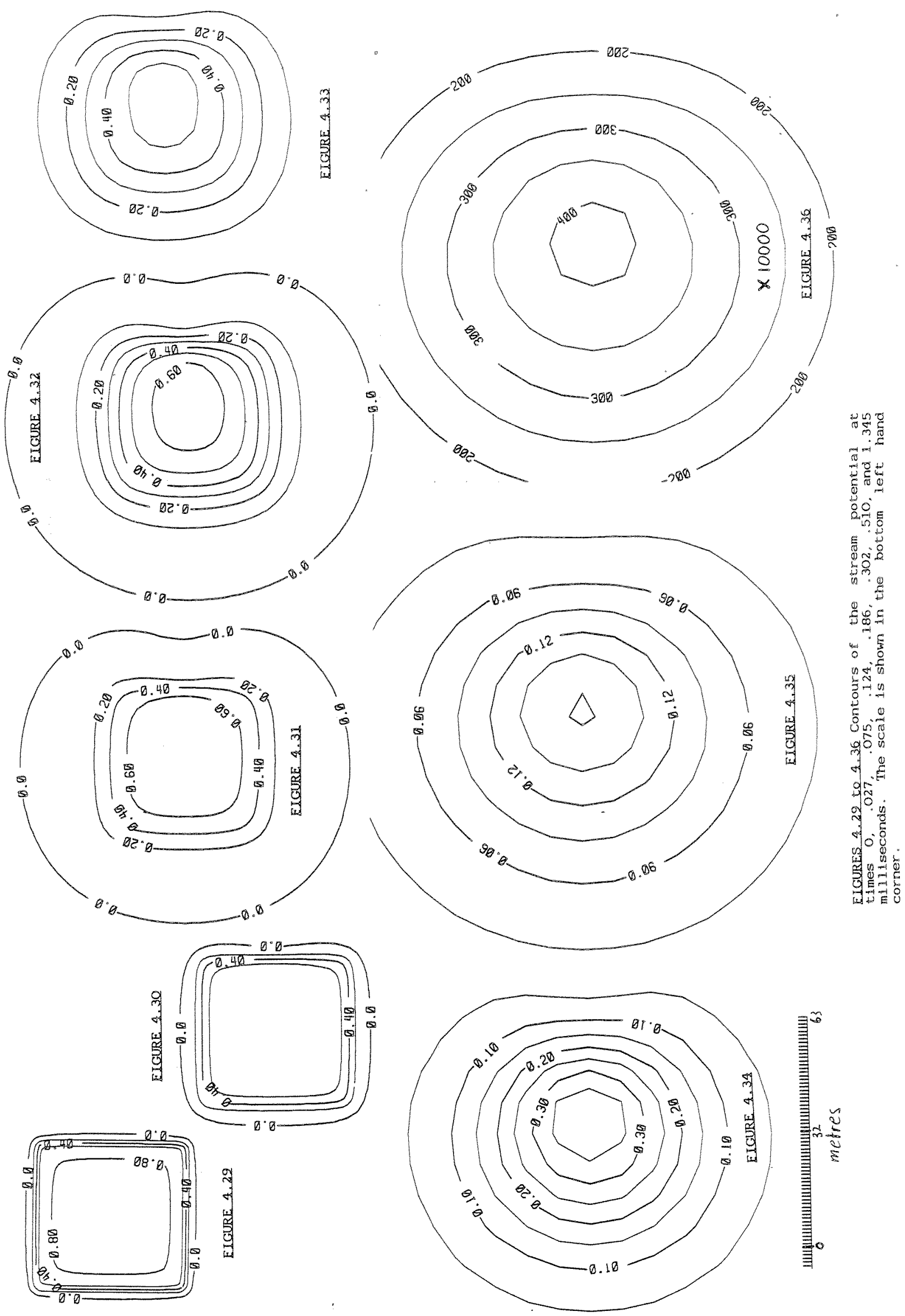




\subsubsection{Internal Consistency Checks}

\subsubsection{Grid spacing}

It is not possible to do unambiguous convergence tests with the program, as it is difficult to describe the inhomogeneity identically in cases of differing grid size. Also, the grid size has upper and lower bounds placed on it to so as to ensure that the initial fields will be adequately represented in the wavenumber domain. The decay of the field for the resistive case discussed above at the point $(22,0)$, is shown for five different grid spacings on figure 4.37 ( $\log$-Iin) and figure 4.38 $(\log -\log )$. The ' $\mathrm{T}$ 's on the log-log plots trace negative values which have been made positive for log plotting. In each case the inhomogeneity is 20 metres by 20 metres, the number of time steps per grid expansion is 15, and the grid is 128 nodes by 128 nodes. The factors which vary from graph to graph are tabulated on table 4.1. The units are metres and the origin is always at the centre of the loop. The grid spacing is the critical factor but the left edges of the grid and inhomogeneity should ideally be the same.

\begin{tabular}{|c|c|c|c|}
\hline $\begin{array}{l}\text { Graph } \\
\text { label }\end{array}$ & $\begin{array}{l}\text { Grid } \\
\text { spacing }\end{array}$ & $\begin{array}{l}\text { Ieft hand } \\
\text { edge of grid }\end{array}$ & $\begin{array}{l}\text { left edge } \\
\text { of inhomo. }\end{array}$ \\
\hline $\begin{array}{l}1 \\
2 \\
3 \\
4 \\
5\end{array}$ & $\begin{array}{l}.83333 \\
.90909 \\
1.0000 \\
1.1111 \\
1.2500\end{array}$ & $\begin{array}{l}-53.3333 \\
-58.1818 \\
-64.0000 \\
-71.1111 \\
-80.0000\end{array}$ & $\begin{array}{l}10.8333 \\
10.9090 \\
11.0000 \\
11.1111 \\
11.2500\end{array}$ \\
\hline
\end{tabular}


Check point $(22,0)$ - grid size varies

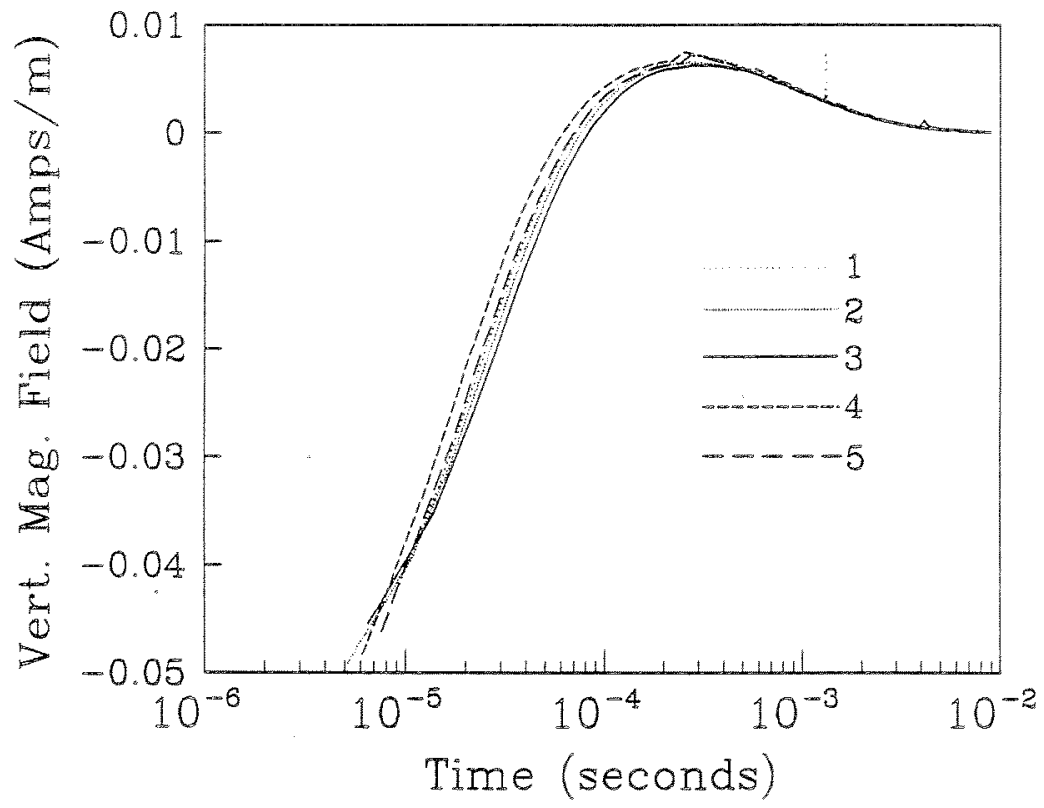

EICURE 4.37 A $10 \mathrm{~g}-1$ inear plot of the vertical magnetic field at $(22,0)$ for a $40 \mathrm{~m}$ by $40 \mathrm{~m}$ loop centred at $(0,0) 1$ metre above the sheet plotted as a function of time using different grid sizes. In each case the grid has 128 by 128 points but the distance between nodes is .3333.9090. points, but the distance between nodes is $.3333,1.9090$. respectively

Check point $(22,0)$ - grid size varies

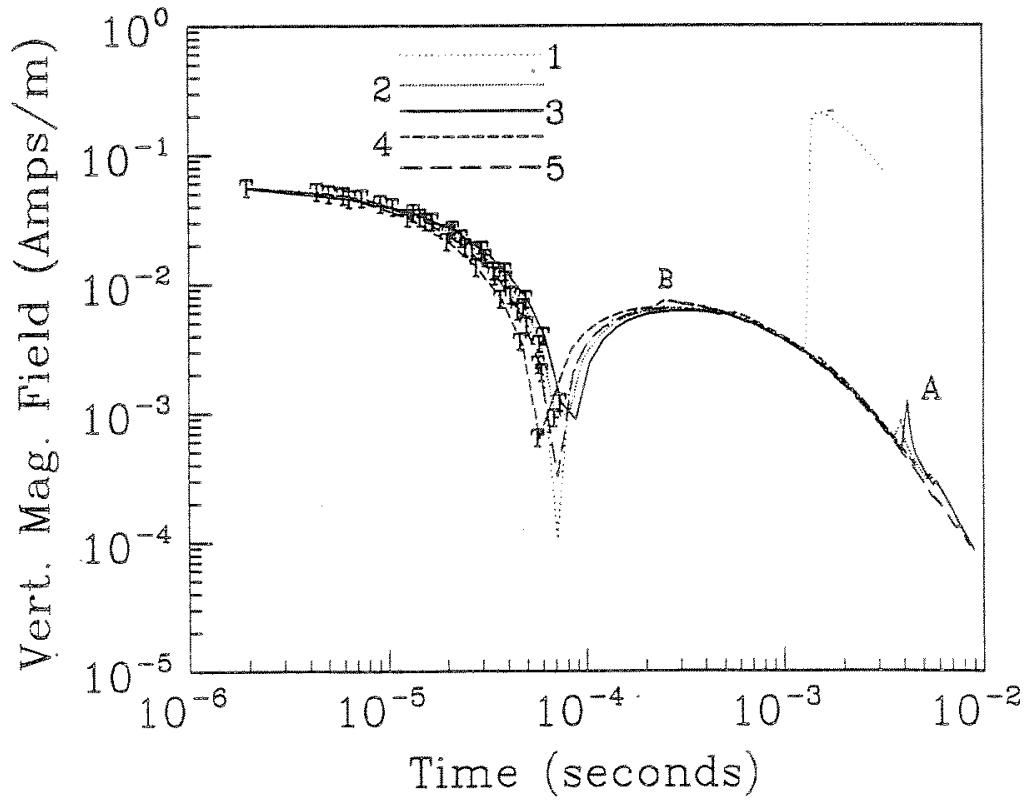

FIGURE 4.38 A log-log plot of the vertical magnetic field at (22,0) for a $40 \mathrm{~cm}$ by $40 \mathrm{~m}$ loop centred at $(0.0) 1$ metre above (22.0) for a $40 m$ by $40 m$ loop centred at $(0,0)$ metre above
the sheet plotted as a function of time using different grid
sizes. In each case the grid has 128 by 128 points, but the sizes. In each case the grid has 128 by 128 points, but the

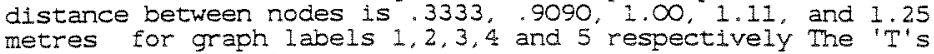
trace negative values. 
As can be seen from both figures 4.37 and 4.38 the time at which the zero crossing passes the check point varies from $70 \mu \mathrm{s}$ to $90 \mu \mathrm{s}$. This is not an insignificant error however it can be explained. Eirstly, the fact that the grid starts and finishes in different places may have some effect. Also, the fact that the inhomogeneity starts on similar but silghtly different positions is also likely to have an effect. Finally the method by which the value of the vertical magnetic field at $(22,0)$ is calculated may also result in some error. The point $(22,0)$ does not always lie on a point of discretization, so a linear interpolation of the surrounding four points was done to obtain the value of the field at this point. This may not be an accurate approximation at early times, however the linear interpolation will result in a consistent error-which is seen on the plots. If the values used to approximate the field at $(22,0)$ are consistently in error by a small amount, then this will result in a significant change in the zero crossing of the vertical field. When the fields vary less rapidly at late delay times the approximation will become better, and this is observed. The spurious 'bumps' (labelled ' $A$ ' and ' $B$ ' on the log-log plot) have occurred before an expansion (B) or a forced grid expansion (A). After the expansion the results appear to have returned to their 'normal' values, except in the case of the graph labelled ' 1 ' which departed from the normal solution so much that it was unable to recover. These instabilities can be overcome by filtering the field.

It would seem from the cases shown that the most stable cases are those with the largest grid size. A good guideline in selecting the grid spacing would therefore be to select the 
largest possible grid spacing which has a tolerable aliasing error.

\subsubsection{Iime step size}

In an attempt to determine the effect of the time step size on the solutions, the number of time steps between grid expansions was varied. Essentially this amounts to varying the time step size, as the times of the grid expansions are approximately constant. The model used in the test is that of the graph labelled ' 2 ' on figures 4.37 and 4.38 . The number of steps between grid expansions used is 9,11,13,15 and 17. These are shown on figure 4.39. Again the ' $T$ 's represent negative values. It would seem that the step size has little effect on the accuracy of the solution prior to the solution blowing up. The effect of a varying step size is to alter the delay time at which the solution becomes unstable. This time is obvious from the plots of the fields in the space domain.

Again the instabilities can be avoided if $H_{z}^{i}$ is filtered, but this increases the computation time slightly, and smoothes the solution. 


\section{Check point $(22,0)$ - step size varies}

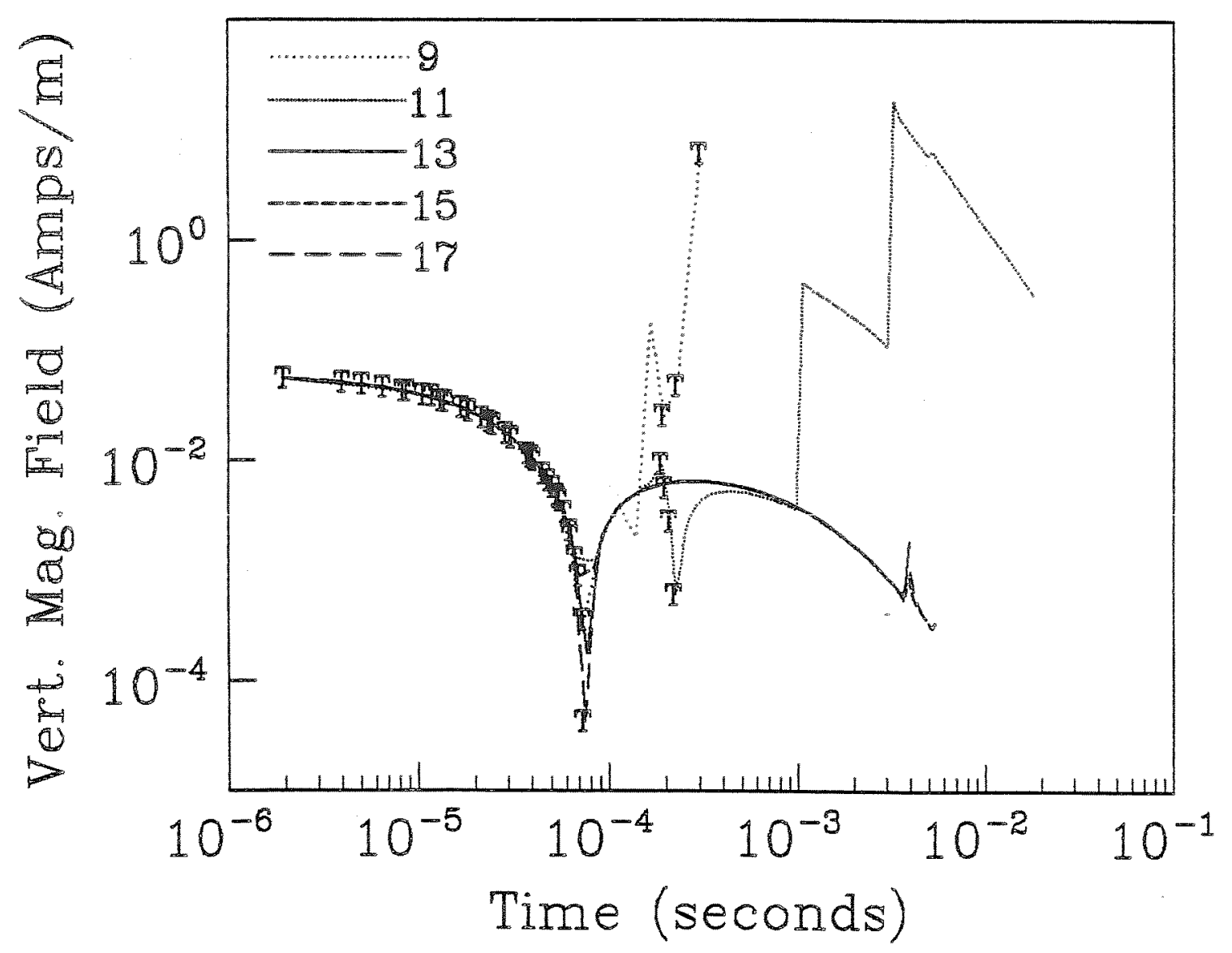

EIGURE 4.39 A log-log plot of the vertical magnetic field at $(22,0)$ for a $40 m$ by 400 loop centred at $(0,0) 1$ metre above the sheet plotted as a function of time using different time steps. The number of steps between grid expansions are 9.11,13,15 and 17. These are plotted on the figure with the line types shown. The ' $T$ 's trace negative values. 


\subsection{CHAPTER 5 - CONCLUSION}

The aim of this thesis was to model the electromagnetic response of a thin sheet with a gently varying conductivity-thickness product ( $\sigma t)$. The sheet is situated in free space. Responses have been obtained in the frequency domain, and in the time domain.

The frequency domain case gives physically reasonable results, however computer storage capabilities restrict the number of wavenumbers which can be used to represent the field to 441 or less. This means that the fields to be studied must vary in such a manner such that they can be described with low wavenumber components. Whenever a new frequency of excitation or conductivity structure is to be solved for the discretization grid must be re-adjusted, and the matrix inverted again.

The advantage of the frequency domain method of solution is that once a solution matrix has been inverted, the matrix can be stored and the response for any number of source fields can be found by matrix multiplication. A profile for a moving transmitter E.M. system over a certain conductivity structure can therefore be computed cheaply. This method of solution is therefore particularly suited to obtaining frequency domain airborne E.M. responses.

Time domain methods are utilized more frequently than frequency domain methods in areas where the conductivity structure is most likely to be made up of only a conducting overburden. These methods usually involve large transmitter loops which are laid close to the ground. At early delay times the fields are concentrated around the loop, and thus high 
spatial variations will be present. This means high wavenumber components are required to describe the fields. Therefore the methods which have been described for the frequency domain case cannot be applied. An algorithm has been developed which steps the fields through time in doing so it uses all the wavenumber components present. The response at late delay time will have a dependence which is best described by low wavenumber components. To adequately describe the fields at late delay times, more low wavenumber information must be included. As a discrete Fourier transform is used, the fields must be sampled with equally spaced sample points. The easiest solution is to double the grid size occasionally. This is the time domain analogue of using different grid spacings for different frequencies.

Eor the variable conductivity problem, the decaying fields will have a 'multimodal' wavenumber distribution. Conductive areas in the sheet will result in some of the high wavenumbers decaying more slowly. Instabilities result at late delay times when the low wavenumbers dominate. By filtering or forcing a grid expansion to retain stability the high wavenumber information relevant to the more conductive areas is thrown away. The solution is thus forced towards the uniform thin sheet solution. A trade off situation exists. Either the solution can be calculated with no approximation up to the latest delay time that the method is stable, or a stable solution can be obtained for all delay times. The stable solution has been spatially smoothed. 
The program takes about 2.5 minutes of cpu time per iteration on a VAX 11/750. Typically 70 time steps may be required to cover the full decay of the rields, thus 3 hours are normally required to time step the fields.

The method uses the fast Fourier transform (EET) to calculate the horizontal component of the magnetic field from the vertical components. This is one of the most time consuming aspects of the program, as virtual memory space is used. If this could be sped up, then the program would be quicker. Using an array processor to calculate the FET may also provide significant savings.

The results obtained from the programs are encouraging, and they now allow the response of a laterally varying conductive overburden to be found. Variable conductivity sheet solutions can now be recognized, and this will aid in distinguishing the effect of overburden from the effect of buried conductors (ore bodies).

An example of a body which could be modelled by such methods as those described here is the weathered zone associated with a kimberlite pipe. 


\section{BIBLIOGRAPHY}

Barringer A.R. (1962) 'A New Approach to Exploration - The INPUT Airborne Electrical Pulse Prospecting System' Mining Congress Journal; Vol 48. no 10 p49-52.

Bracewell R.N. (1978) 'The Eourier Transform and its Applications' McGraw Hill (New York) Electrical and Electronic Engineering Series

Das N.C. and Verma S.K. (1982) 'Electromagnetic response of an arbirtarily shaped three dimensional conductor in a layered earth - Nunerical Results', Geophysical Journal Roy. Ast. Soc. Vol 68 p55-66.

Dyck A.V., Bloore M. and Vallee M.A. (1980) "User Manual for Programs PLATE and SPHERE" Research in Applied Geophysics, Geophysics Lab. University of Toronto

Gaur V.K. and Verma O.P. (1973) 'Enhancement of Electromagnetic Anomalies by a Conducting overburden II'. Geophysical Prospecting Vol 21 p159-184

Geyer R.G. and Wait J.R. (1978) 'Electromagnetic Fields of a Vertical Magnetic Dipole above a Laterally Inhomogeneous Thin layer of Conductive Overburden' Pageoph, Vol 116. p181-197 
Grant E.S. and West G.F. (1965) 'Interpretation Theory in Applied Geophysics" McGraw-Hill. Book Coy. Inc. N.Y.

Greenfield R.J. (1971) 'The Electromagnetic Response of a conducting disk for use in AEMAG Interpretation', Geophysics, Vol 36, p723-738.

Gupta O.P., Joshi M.S. and Negi J.G., (1980) 'Scale model Electromagnetic Response to Inline and Broadside Systems at Skew Traverses of dipping half planes Embedded in a Conducting Host Rock.' Geophysical Prospecting. Vol 28. p119-134.

Lajoie J.J. and West G.E. (1976) "The electro magnetic response of a Conductive inhomogeneity in a layered earth'. Geophysics Vol 41 p1133-1156

Lapidus L. and Pinder G.E. (1982) "Numerical Solutions of Partial Differential Equations in Science and Engineering" John Wiley and Sons (New York)

Lowrie $W$. and West G.F.' (1965) 'The effect of a conducting overburden on electromagnetic prospecting measurements' Geophysics Vol 30 p624-632

Malilik K. (1972) 'Conducting sphere in Elecromagnetic INPUT field'. Geophysical Prospecting. Vol 20 p293-303

MCKirdy D.MCA. and Weaver J.T. (1984)' Induction in a thin sheet of variable conductance at the surface of a 
stratified earth - I. Two dimensional theory'. Geophys J. R. astr. Soc. 78, 93-103

Mesinger E. and Arakawa A. (1976) 'Numerical Methods used in Atmospheric Models - Volume 1', Global Atmospheric Research Programme (GARP) Series No. 17

Nelson P.H. and Morris D.B. (1969) 'Theoretical Response of a Time Domain, Airborne, Electromagnetic . System." Geophysics Vol 34 p729-738

Oristaglio M.L. and Hohmann G.W. (1984) 'Diffusion of electromagnetic fields into a two-dimensional earth: A finite-difference approach'. Geophysics. Vol 49. p870-894.

Palacky G.J. (1975) 'Interpretation of INPUT AEM Measurements in Areas of Conductive Overburden". Geophysics Vol 40. $\mathrm{p} 490$

Price A.T. (1949) "The induction of electric currents in non-uniform thin sheets and shells.' Quart J. Mech. and Appl. Math. Vol. III, Pt 3, p283-310.

Kanasewich E.R. (1981) 'Time sequence analysis in Geophyics'. Univ. of Alberta Press (Edmonton) 3rd Edition.

Knight J.M. and Raiche A.P. (1982) 'Transient Electromagnetic Calculations using the Gaver-Stehfest Inverse Laplace transform Method' Geophysics, Vol 47, p47-50 
Richtmeyer R.D. and Morton K.W. (1967) 'Difference Methods for Initial-Value Problems'. Interscience Publishers (John Wiley and Sons N.Y.) Second Edition.

Singh S.K. (1973) 'Electomagnetic Transient Response of a Conducting Sphere embedded in a Conductive Medium', Geophysics Vol 38 p864-893

Taylor J.R. (1982) 'An Introduction to Error Analysis. The study of Uncertainties in Physical measurements." University Science Books, Mill Valley California.

Telford W.M., Geldart L.P., Sheriff R.E., Keys D.A. (1976) 'Applied Geophysics.' Cambridge University Press, Cambridge.

Verma S.K. (1972) 'Quasi-static Time Domain Electromagnetic Response of a Homogeneous Conducting Infinite Cylinder'. Geophysics Vol 37 p92-97.

Wait J.R. (1982) 'Geoelectromagnetism'. Academic Press, N.Y.

Wannamaker P.E., Hohmann G.W. and SanEilipo W.A. (1984) 'Electromagnetic Modelling of three dimensional bodies in Layered Earths using integral Equations.' Geophysics Vol 49. $\mathrm{p} 60-74$

Ward S.H. (1967) 'The Electromagnetic Method", Mining Geophysics Vol II. The Society of Exploration 
Geophysicists. p224-372

Young J.A. (1968) 'Comparative Properties of some time differencing schemes for linear and non-linear oscillations". Monthly Weather Review. Vol 96. p357-364. 